\title{
Mecanismos de proteção perante a vitimização por pares e a discriminação
}

\author{
Daniela Fonseca Freitas ${ }^{1}$, Susana Coimbra ${ }^{1}$, Anne Marie Fontaine ${ }^{1}$ \& Edna Maria \\ Marturano $^{2}$ \\ ${ }^{1}$ Centro de Psicologia da Universidade do Porto; Faculdade de Psicologia e de Ciências de Educação da \\ Universidade do Porto \\ ${ }^{2}$ Faculdade de Medicina de Ribeirão Preto da Universidade de São Paulo
}

\begin{abstract}
Resumo: A vitimização por pares e a discriminação, nomeadamente de minorias étnicas e sexuais, têm um efeito negativo no funcionamento psicossocial, mas diversos estudos começam a abordar a resiliência perante estas adversidades. Neste sentido, o objetivo deste estudo foi identificar mecanismos protetores da saúde mental e do desajustamento externo na presença destas formas de violência social. 0 estudo foi conduzido numa amostra de 2975 de estudantes do ensino secundário português, dos quais 245 identificaram-se como negros e 84 como gay, lésbicas ou bissexuais. Os mecanismos de proteção avaliados incluem baixos níveis de personalidade ansiosa, competências de empatia, as estratégias de coping usadas, o otimismo familiar e as relações com as figuras parentais. 0 resultado das regressões revela que a identificação racial e a orientação sexual moderam a influência das diferentes formas de violência e dos diferentes mecanismos de proteção, sugerindo suporte à abordagem socioecológica da resiliência.
\end{abstract}

Palavras-chave: Resiliência; Mecanismos de proteção; Jovens negros; Jovens gay, lésbicas e bissexuais.

Protection mechanisms in the face of peer victimization and discrimination: Peer victimization and discrimination, namely of ethical and sexual minorities, have a negative effect on psychosocial functioning but several studies begin to address the resilience to these adversities. In this sense, the objective of this study was to identify protective mechanisms of mental health and external maladjustment in the presence of these forms of social violence. The study was conducted with a sample of 2975 Portuguese high school students, of which 245 identified as black and 84 as gay, lesbian or bisexual. The protection mechanisms considered include low levels of anxious personality, empathy abilities, the coping strategies used, familial optimism and the relationships with the parental figures. The regression results show that racial identification and sexual orientation moderate the influence of the different forms of violence and the different protection mechanisms, suggesting support to a socio-ecological approach of resilience.

Keywords: Resilience; Protection mechanisms; Black youths; Gay, lesbian and bisexual youths.

A vitimização por pares e a discriminação representam processos sociais que ameaçam o funcionamento psicossocial ótimo das crianças, jovens e adultos, condicionando o sentimento de pertença das pessoas (Richman \& Leary, 2009). As consequências psicológicas de ambas as formas de rejeição social são similares. Estudos apontam que ter sofrido vitimização por parte dos pares ou bullying está associado a aumento da sintomatologia psicopatológica internalizada (e.g., saúde mental generalizada, depressão, ansiedade) e externalizada (e.g., agressão, delinquência, insucesso escolar) (Hawker \& Boulton, 2000; Reijntjes, Kamphuis, Prinzie, Boelen, Van Der Schoot, \& Telch, 2011; Reijntjes, Kamphuis, Prinzie, \& Telch, 2010; Rivers \& Cowie, 2006). Para além disso, a vitimização pelos pares está também associada à diminuição de mecanismos internos que permitem uma adaptação bem-sucedida perante os desafios da vida (tais como a autoestima, a autoeficácia, a persistência, a perceção de suporte, ou otimismo) e a alterações fisiológicas, nomeadamente a alterações na atividade no eixo hipotálamo-pituitária-adrenal (HPA) que controla a libertação do cortisol (Fullchange \& Furlong, 2016; Hawker \& Boulton, 2000; Takizawa, Maughan, \& Arseneault, 2014). Também os estudos sobre a perceção de discriminação indicam

\footnotetext{
${ }^{1}$ Endereço para correspondência: Faculdade de Psicologia e de Ciências da Educação da Universidade do Porto, Rua Alfredo Allen, 4200 -135 Porto, Portugal. E-mail: daniela.ffreitas@gmail.com / susana@fpce.up.pt. Este trabalho foi financiado por duas bolsas atribuídas a Daniela Freitas, uma de doutoramento pela FCT - Fundação para a Ciência e Tecnologia (SFRH/BD/79575/2011) e outra pelo programa de Bolsas USP Internacional para alunos de Instituições de Ensino Superior Estrangeiras na USP (2014.1.3765.1.1). Adicionalmente, este trabalho foi financiado pelo Centro de Psicologia da Universidade do Porto, Fundação para a Ciência e a Tecnologia (FCT UID/PSI/00050/2013) e pelo FEDER através do programa COMPETE 2020 (POCI-01-0145-FEDER-007294).
} 
que a mesma está associada a um aumento da depressão, ansiedade e stress pós-traumático e a uma diminuição da autoestima, da perceção de controlo ou mestria, da satisfação com a vida e alguns indicadores da saúde física (para estudos de revisão consultar Paradies, Bem, Denson, Elias, Priest, Pieterse, ..., \& Gee, 2015; Pascoe \& Richman, 2009; Schmitt, Branscombe, Postmes, \& Garcia, 2014). No presente estudo, iremos focar-nos em grupos alvo de dois preconceitos distintos, o racismo e a homofobia.

\section{A vitimização por pares e a discriminação como mecanismos de risco para o desenvolvimento}

A vitimização entre pares tem sido definida como uma forma de abuso, pela qual uma criança ou adolescente é frequentemente alvo de agressão pelos seus pares (Kochenderfer \& Ladd, 1996). Já a discriminação pode ser definida como um tratamento diferenciado e negativo de pessoas com base na sua pertença a um determinado grupo social (Dovidio, Major, \& Crocker, 2000). Ainda que sejam fenómenos distintos, a vitimização por pares e a discriminação parecem, em parte, coexistir, sendo que a vitimização entre pares baseada no preconceito é designada de bullying discriminatório (Elamé, 2013). Este é definido como "uma forma de abuso e vitimização associada à incapacidade/deficiência, sexo, origem étnica, orientação sexual e religião que ocorre repetidamente no decorrer do tempo por um ou mais pares" [tradução própria] (Elamé, 2013, p. 28). Neste sentido, alguns estudos apontam o desvio à norma como a motivação mais frequente para a perpetração de agressões entre pares (Freire, Simão, \& Ferreira, 2006; Strohmeier, Kärnä, \& Salmivalli, 2011; Thornberg, 2010). Adicionalmente, Carrera-Fernández, Lameiras-Fernández, Rodríguez-Castro e Vallejo-Medina, (2013) observaram uma associação entre as atitudes positivas perante as agressões entre pares e a manifestação de preconceitos, nomeadamente o sexismo e a homofobia.

São várias as características pessoais que podem ser estigmatizadas numa determinada cultura. Em Portugal, segundo os dados do Eurobarómetro, os tipos de discriminação mais comuns parecem ser aqueles que têm por base a orientação sexual (69\% dos participantes concorda que a discriminação de minorias sexuais é comum), a identidade de género (65\%), a deficiência (65\%) e a origem étnica (64\%) (European Commission, 2015). 0 preconceito em relação a estes e outros grupos parece manifestar-se logo na infância (Cunha, 2014; França \& Monteiro, 2004), sendo por isso de esperar que, no contexto escolar português, se observem atos de discriminação racista e homofóbica.

Estudos têm demonstrado que o bullying discriminatório tem um impacto mais nocivo do que a vitimização não baseada no preconceito, quer a nível interno (e.g., sintomatologia depressiva, confiança que têm nos outros, otimismo, controlo emocional), quer a nível externo (e.g., nas relações com os pares e com os adultos, no comportamento escolar e consumo de substâncias) (Gradinger \& Strohmeier, 2015; Hunter, Durkin, Heim, Howe, \& Bergin, 2010; Russell, Sinclair, Poteat, \& Koenig, 2012). O estudo de Hunter et al. (2010) sugere ainda que as crianças que são vítimas de bullying discriminatório, no caso discriminação étnica, demonstram menor perceção de controlo sobre os comportamentos abusivos dos seus colegas. Deste modo, os jovens de grupos minoritários e alvo de discriminação estão em maior risco de desajustamento do que os seus pares, pois não só é mais provável que sejam mais frequentemente vítimas, como é provável que esta vitimização se associe com uma maior magnitude à perturbação psicológica e social (Gradinger \& Strohmeier, 2015; Hatzenbuehler, 2009; Hunter et al., 2010; Russell et al., 2012). Deste modo, é pertinente o estudo centrado no ajustamento dos jovens portugueses alvo de preconceito, com os jovens de minorias étnicas e sexuais.

Abordando primeiramente os jovens que poderão ser alvo de preconceito racista ou xenófobo, estudos em contexto europeu revelam uma maior prevalência da vitimização por pares junto de jovens imigrantes ou filhos de imigrantes (e.g., del Barrio, Martin, Montero, Gutiérrez, Barrios, \& Dios, 2008; Strohmeier et al., 2011). Os estudos sobre o bullying ou a vitimização por pares junto de jovens de minorias étnicas em Portugal são raros (Carvalhosa, Moleiro, \& Sales, 2009). Dos poucos estudos observados, dois apontam que, quando controlado o estatuto socioeconómico, as crianças de origem imigrante africana ou brasileira apresentam níveis similares de provocação por parte dos seus pares (avaliada de forma genérica) aos apresentados pelas crianças autóctones (Gaspar, Matos, Ribeiro, \& Leal, 2010; Matos, Gonçalves, \& Gaspar, 2004). Já no estudo multinacional coordenado por Elamé (2013) reporta-se uma maior frequência na vitimização dos jovens de minorias étnicas (sobretudo jovens de etnia cigana e imigrantes do leste da Europa) em Portugal. No que concerne à discriminação étnica, o estudo não comparativo conduzido por Neto (2010a), no qual os jovens foram questionados se foram alvo de insultos (e outros atos negativos) devido à sua origem étnica, revela que cerca de $19 \%$ dos jovens de comunidades imigrantes (sobretudo jovens afro-lusitanos) sentem-se discriminados com frequência. Assim, estudos com metodologias diferentes revelam resultados que se complementam. Enquanto a frequência de atos de vitimização entre pares entre jovens com ascendência imigrante parece ser, nalguns 
casos, similar à dos jovens autóctones, uma parte de jovens com ascendência imigrante alega que a sua etnia é motivo para serem tratados de forma negativa.

Os jovens de minorias sexuais constituem o outro grupo social em estudo, pois são alvo provável de preconceito homofóbico e frequentemente vítimas de agressões. A violência que estes sofrem tem sido reportada em contexto escolar, familiar e em outros contextos sociais ao longo da vida (Fedewa \& Ahn, 2011; Katz-Wise \& Hyde, 2012). Alguns dados portugueses apontam que $42 \%$ de jovens que se identificam como sendo lésbica, gay ou bissexual (LGB) viveram situações de bullying homofóbico (António, Pinto, Pereira, Farcas, \& Moleiro, 2012).

\section{Resiliência, equilíbrio entre o risco e proteção}

0 desenvolvimento psicossocial é um produto do sujeito em contínua interação com o seu contexto. Neste âmbito, a resiliência pode ser definida como o resultado de um processo desenvolvimental dinâmico no qual se observa uma adaptação positiva na presença de adversidade significativa (Masten \& Wright, 2010). Deste modo, o estudo da resiliência implica sempre a consideração do risco e do ajustamento. Entende-se por risco qualquer condição que esteja associada à ocorrência de desequilíbrios e/ou distúrbios psicossociais, constituindo, por isso, uma ameaça ao funcionamento "adequado" ou "normal" da pessoa. Considerando os riscos para o desenvolvimento que representam as situações de violência entre pares, assim como a discriminação, recentemente, a investigação tem-se focado não só na vitimização entre pares e nas suas consequências negativas, mas também no processo de resiliência e mecanismos de proteção perante esta forma de violência (e.g., Averdijk, Eisner, \& Ribeaud, 2014; Bowes, Maughan, Caspi, Moffitt, \& Arseneault, 2010; Freitas, Coimbra, Marturano, Marques, Oliveira, \& Fontaine, 2017; Hemphill, Tollit, \& Herrenkohl, 2014; McVie, 2014, Ttofi, Bowes, Farrington, \& Lösel, 2014; Vassallo, Edwards, Renda, \& Olsson, 2014) e que favorecem o ajustamento positivo de pessoas que são membros de grupos alvo de discriminação (e.g., APA Task Force on Resilience and Strength in Black Children and Adolescents, 2008; Eisenberg \& Resnick, 2006; Gaylord-Harden \& Cunningham, 2009; Juang \& Alvarez, 2010; Murdock \& Bolch, 2005; Neto, 2009, 2010a, 2010b; Ryan, Russell, Huebner, Diaz, \& Sanchez, 2010; Saewyc, 2011; Szalacha, Erkut, Coll, Fields, Alarcón, \& Ceder, 2003).

Os estudos demonstram que diversas dimensões, a nível individual, familiar ou comunitário, distinguem as pessoas consideradas resilientes daquelas que não manifestam um ajustamento tão positivo em condições de adversidade. Estas dimensões que diferenciam os perfis de ajustamento têm sido reconhecidas como mecanismos de proteção do ajustamento na presença do risco (Coimbra \& Fontaine, 2015; Fergus \& Zimmerman, 2005; Luthar, Cicchetti, \& Becker, 2000; Masten, 2001; Masten \& Wright, 2010; Rutter, 2005, 2013; Ungar, 2013). Os mecanismos individuais no processo de resiliência têm-se focado sobretudo nas competências intraindividuais, tais como a autoestima, competências sociais, traços de personalidade, o otimismo, nas estratégias de coping utilizadas e, cada vez mais, na suscetibilidade diferencial de cada indivíduo às influências do meio (Coimbra, 2008; Dumont \& Provost, 1999; Fergus \& Zimmerman, 2005; Herman-Stahl \& Petersen, 1996; Masten \& Tellegen, 2012; Rutter, 2005, 2013; Ungar, 2013; Vanderbilt-Adriance \& Shaw, 2008; Werner, 1993). Adicionalmente, as dimensões do contexto familiar são importantes para favorecer o desenvolvimento positivo, especialmente em situações de adversidade. Os mecanismos de proteção familiares podem variar de recursos materiais e instrumentais, tais como a presença de um cuidador que fornece abrigo e nutrição, a recursos emocionais, que incluem relações parentais de proximidade e afeto positivo ou práticas de monitorização (Fergus \& Zimmerman, 2005; Vanderbilt-Adriance \& Shaw, 2008). Já a nível comunitário, os estudos têm destacado a qualidade do local de residência, o ambiente escolar ou a disponibilidade e $o$ acesso aos bens/recursos institucionais (sistema de saúde, programas de apoio) (Vanderbilt-Adriance \& Shaw, 2008; Ungar, 2013).

De acordo com a perspetiva socioecológica defendida por Ungar (2013), a manifestação de resiliência deve-se à existência recursos (sociais, culturais e físicos) providenciados pelo contexto e à capacidade de as pessoas acederem a estes de forma a manterem o seu bem-estar. Ungar (2004) defende que é esta negociação com os recursos que são específicos do seu contexto, que permite que as pessoas se definam como saudáveis apesar das adversidades que viveram. De acordo com o princípio da relatividade cultural, a manifestação da resiliência ocorre de acordo com o contexto cultural, sendo que muitas das estratégias usados pelos jovens para lidar com as adversidades são ilustrativas das oportunidades que o contexto lhe fornece (Ungar, 2004, 2011).

A especificidade do contexto justifica também porque é que alguns mecanismos são promotores do ajustamento em determinadas condições e não em outras, observando-se assim uma influência diferencial. No âmbito do estudo da vitimização entre pares, Bowes et al. (2010) observaram que apesar de o carinho da mãe e o ambiente familiar positivo ser promotor, de uma forma geral, de melhor ajustamento emocional e comportamental, o seu efeito positivo era maior para as crianças que tinham 
sofrido bullying. A especificidade do contexto é também relevante no que diz respeito ao estatuto minoritário e/ou estigmatizado. Pearson e Wilkinson (2013), numa amostra representativa dos adolescentes dos EUA, observaram que uma maior perceção de proximidade aos pais (e.g., carinho, satisfação, comunicação) estava associada a um menor consumo de álcool por parte das adolescentes. Porém, este efeito protetor era menor nas raparigas lésbicas ou bissexuais, comparativamente às heterossexuais. A proximidade aos pais revelou também estar associada a menores níveis de depressão nos rapazes, só que neste caso a influência protetora exercida era maior para os adolescentes gay ou bissexuais. Este estudo revelou ainda que uma maior frequência de atividades de lazer com a família estava associada a menor consumo de drogas e fugas de casa nos rapazes heterossexuais, porém nos jovens gay e bissexuais foi observada uma associação no sentido oposto. Estes são alguns dos estudos que sugerem que um mesmo mecanismo tem um impacto diferencial em função do contexto na qual se manifesta a adversidade, sustentando a abordagem socioecológica da resiliência. Deste modo, quando procuramos explorar o processo de resiliência junto de jovens de grupos minoritários e discriminados, a adoção de uma perspetiva ecológica no estudo dos mecanismos de risco e de proteção é fulcral, até porque o próprio estigma e a manifestação do preconceito são indissociáveis do contexto.

Considerando o exposto anteriormente, este estudo procurou, junto de uma amostra de adolescentes, observar em que medida a vitimização por pares e a perceção de discriminação, assim como alguns mecanismos de proteção (individuais e familiares) influenciam a saúde mental e se associam ao (des)ajustamento externo (Luthar et al., 2000). Mais precisamente, avaliamos os níveis de personalidade ansiosa, das competências empáticas e do uso de estratégias de coping (a nível individual), do otimismo familiar e de qualidades das relações com a mãe e com o pai (ou outros/as cuidadores/as). Considerando a ecologia do processo de resiliência, esta exploração de associações incluiu a diferenciação dos adolescentes de acordo com a identificação racial e a orientação sexual.

\section{MÉTODO}

\section{Amostra e procedimento de recolha de dados}

A amostra é constituída por 2975 adolescentes com idade média de 16.6 anos ( $D P=1.27)$. Esta é equilibrada em termos de sexo (54.2 \% são do sexo feminino) e nível de qualificações parentais, sendo que $31.1 \%$ dos participantes têm pais com o ensino básico obrigatório, $38.0 \%$ têm pelo menos um educador com o ensino secundário, e 30.9\% têm pelo menos uma figura parental com formação universitária. A maioria dos participantes estuda em cursos científico-humanísticos (81.6\%). Cerca de 92\% nasceram em Portugal, em termos de descrição étnica/racial, 89.1\% são brancos/caucasianos, 8.5\% negros, $1.4 \%$ mestiços e 1\% com outra descrição (e.g., etnia cigana ou asiáticos). Na descrição da orientação sexual, $97.2 \%$ descrevem-se como heterossexuais, $2.4 \%$ como bissexuais e $0.5 \%$ como gays ou lésbicas.

Este estudo foi aprovado pela Comissão de Ética da Faculdade de Psicologia e de Ciências da Educação da Universidade do Porto, pela Comissão Nacional de Proteção de Dados (protocolo 355/2013) e recebeu permissão do Ministério da Educação (processo 0352400001) para ser conduzido em estabelecimentos de ensino públicos em Portugal. Colaboraram no estudo 24 escolas (63.4\% das escolas selecionados aleatoriamente e contactadas) em cinco cidades das áreas metropolitanas de Lisboa e Porto e a recolha de dados ocorreu maioritariamente durante o ano académico de 2013/2014. Os encarregados de educação e alunos foram informados que o principal objetivo do estudo era explorar como os jovens lidam com situações sociais negativas, foram ainda esclarecidos sobre o procedimento e a confidencialidade dos dados e deram o seu consentimento à participação. Os participantes preencheram o questionário em contexto de sala de aula na presença da primeira autora deste estudo. 0 preenchimento do questionário demorou, em média, 35/45 minutos e a taxa de participação foi de 94.7\%.

\section{Instrumentos}

Os instrumentos incluídos no questionário versaram sobre dimensões essenciais no quadro teórico da resiliência: (1) indicadores de ajustamento, (2) mecanismos de risco e (3) mecanismos de proteção. Foram também recolhidas informações demográficas gerais.

\section{Indicadores de ajustamento}

Para avaliação da saúde mental, utilizou-se a versão portuguesa do Mental Health Inventory-5 (Ribeiro, 2001; Marques, Pais-Ribeiro, \& Lopez, 2011; Veit \& Ware, 1983). 0 instrumento inclui 5 itens relacionados com a ansiedade, depressão e o bem-estar (e.g., Durante o último mês quanto tempo te sentiste triste e em baixo?), e desta forma, é consonante com a definição de saúde apresentada atualmente pela Organização Mundial de Saúde (WHO, 2014). Os itens foram respondidos numa escala de resposta de 
tipo Likert com 5 pontos (1 - nunca, 5 - quase sempre). A escala demonstrou um bom ajustamento à amostra, $\left[\chi^{2} / \mathrm{gl}=3.91 ; \mathrm{CFI}=0.99 ; \mathrm{RMSEA}=0.03 ; p_{R S M E A}=1.000 ; \mathrm{SRMR}=.007 ; \lambda>.54 ; \mathrm{VEM}>.49 ; \mathrm{CR}>.82\right]$ e um valor de consistência interna muito satisfatório [ $\alpha$ de Cronbach $=.83$ ].

A avaliação do desajustamento externo baseou-se em seis acontecimentos de vida associados a um desempenho negativo em tarefas sociais valorizadas nesta faixa etária: três itens de conduta problemática, nomeadamente problemas com dependências (tabaco, álcool ou outras drogas), ter agredido fisicamente alguém e ter problemas com a polícia ou o sistema judicial; outros três itens de experiências de insucesso escolar, mais precisamente, ter reprovado um ano, ter conflitos graves com os professores e não ter conseguido entrar no curso pretendido (na transição para o ensino secundário os alunos escolhem o tipo de curso e a área que querem frequentar, e.g., cursos profissionais ou científicoshumanísticos). Estes itens fazem parte de um Inventário de Acontecimentos de Vida que foi elaborado com base em outro inventário de acontecimentos de vida (Coimbra, 2008) e de eventos stressantes (Kristensen, Leon, D’Incao, \& Dell'Aglio, 2004). Sendo este instrumento um inventário de acontecimentos e não propriamente uma escala de avaliação de constructos psicológicos, as suas qualidades psicométricas não foram avaliadas mediante análises fatoriais e de consistência interna. No entanto, verificou-se que a soma destes indicadores - perfazendo um índex de 7 pontos ( 0 a 6) - apresentava uma distribuição com propriedades similares à da curva normal, mais precisamente valores de assimetria curtos e abaixo de $1(s k=0.90, k u=0.18)$. Deste modo, este indicador foi utilizado nas análises de regressão linear, pois essas análises são robustas ao não cumprimento dos pressupostos de normalidade e homogeneidade de variâncias (Kline, 1988, como citado em Marôco, 2011).

\section{Mecanismos de Risco}

O risco considerado no presente estudo foi a violência social. Esta foi avaliada com recurso a duas formas de vitimização, uma de vitimização pelos pares (bullying) e outra relativa à perceção de tratamento diferencial negativo (discriminação). Foram utilizados dois itens (apresentados num Inventário de Acontecimentos de Vida): (1) ter sido vítima de bullying e (2) ter sido vítima de discriminação. Em relação a estes dois eventos, os participantes teriam de se pronunciar se alguma vez estas situações aconteceram com eles com base nas seguintes opções de resposta: Nunca aconteceu ou Aconteceu.

Adicionalmente, a vitimização por pares foi avaliada com recurso à versão portuguesa da Peer Victimization Scale (Mynard \& Joseph, 2000; Veiga, 2007). Este instrumento avalia quatro tipos de vitimização; física (3 itens, e.g. Feriram-me fisicamente.), verbal (4 itens, e.g., Gozaram comigo.), social (4 itens, e.g., Tentaram pôr os/as meus/minhas amigos/as contra mim.) e os ataques à propriedade (4 itens, e.g., Roubaram-me alguma coisa.). Os itens foram respondidos numa escala de resposta de tipo Likert com 5 pontos ( 1 - nunca, 5 com muita frequência). As subescalas apresentaram valores de consistência interna satisfatórios $(\alpha>78)$ e a Análise Fatorial Confirmatória (AFC) à estrutura da escala revelou um ajustamento satisfatório à amostra $\left[\chi^{2} / \mathrm{gl}=5.93 ; \mathrm{CFI}=0.97\right.$; RMSEA $=0.05 ; p_{\mathrm{RSMEA}}=.120 ; \mathrm{SRMR}=.34 ; \lambda>$ .484; VEM > .470; CR > .789].

Para avaliar os atos de discriminação crónica utilizou-se a Everyday Discrimination Scale (Williams, Yu, Jackson, \& Anderson, 1997). Os itens foram respondidos numa escala de resposta de tipo Likert com 6 pontos (0 - nunca, 5 - quase sempre (quase todos os dias)). Como este instrumento não se encontrava adaptado ao contexto português, foi realizada uma adaptação do instrumento à amostra do estudo (Freitas, Coimbra, Marturano, \& Fontaine, 2015). A adaptação evidenciou uma estrutura de 2 fatores: Tratamento Injusto (4 itens; e.g., És ameaçado/a ou provocado/a.) e Rejeição Pessoal (4 itens; e.g., $A s$ pessoas agem como se elas fossem melhores do que tu.). Esta estrutura fatorial apresenta índices de ajustamento global satisfatórios $\left[\chi^{2} / \mathrm{gl}=3.42 ; \mathrm{CFI}=0.98 ; \mathrm{RMSEA}=0.04 ; p_{\mathrm{RSMEA}}=.519 ; \mathrm{SRMR}=.317 ; \lambda>\right.$ $.463 ; \mathrm{VEM}>.33$; CR > .65] e valores de consistência interna também satisfatórios [ $\alpha>$. 72].

\section{Mecanismos de proteção}

Os mecanismos de proteção considerados no estudo incluem dimensões do nível individual e do nível familiar. Ao nível individual foram consideradas a personalidade ansiosa, a empatia, bem como a utilização de diversas estratégias de coping. No que concerne à personalidade ansiosa, esta foi avaliada com recurso à versão portuguesa da subescala da Ansiedade do Neuroticism, Extraversion, Openness Personality Inventory Revised (NEOPI-R, Costa \& McCrae, 2000; Lima \& Simões, 2006). Os itens foram respondidos numa escala de resposta de tipo Likert com 5 pontos $(1$ - discordo totalmente, 5 - concordo totalmente). A estrutura unifatorial dos sete itens desta escala (e.g., De vez em quando, vêm-me pensamentos de preocupação à cabeça.) manifestou um ajustamento satisfatório $\left[\chi^{2} / \mathrm{gl}=3.70 ; \mathrm{CFI}=0.99\right.$; RMSEA $=0.03 ; p_{R S M E A}=0.999 ;$ SRMR $\left.=.017 ; \lambda>.47 ; \mathrm{VEM}>.25 ; \mathrm{CR}>.70 ; \alpha=.71\right]$.

Para avaliação da Empatia foi utilizada a versão Portuguesa do Interpersonal Reactivity Index (Davis, 1983; Limpo, Alves, \& Castro, 2010). Duas das quatro subescalas do instrumento foram utilizadas, 
a Tomada de Perspetiva (5 itens; e.g., Quando estou aborrecido/a com alguém, geralmente tento pôr-me no seu lugar por um momento.) e a Preocupação Empática (5 itens; e.g., Quando vejo que se estão a aproveitar de uma pessoa, sinto vontade de a proteger.). Estas subescalas avaliam os processos cognitivos e emocionais perante o estado emocional ou condição de outros. Os itens foram respondidos numa escala de resposta de tipo Likert com 5 pontos ( 1 - discordo totalmente, 5 - concordo totalmente). Na presente amostra, esta estrutura fatorial com as duas subescalas demostrou um ajustamento satisfatório $\left[\chi^{2} / \mathrm{gl}=\right.$ 3.66; $\mathrm{CFI}=0.98 ;$ RMSEA $=0.03 ; p_{\text {RSMEA }}=1.000 ; \mathrm{SRMR}=.021 ; \lambda>.46 ; \mathrm{VEM}>.27 ; \mathrm{CR}>.64 ; \alpha-\mathrm{TP}=.84 ; \alpha-$ $\mathrm{PE}=.64]$.

Para avaliação do uso de diferentes estratégias de coping utilizou-se a versão portuguesa da escala Brief COPE (Carver, 1997; Carver, Scheier, \& Weintraub, 1989; Ribeiro \& Rodrigues, 2004). Este instrumento avalia 14 estratégias de coping em 28 itens (dois itens em cada subescala). Estes foram respondidos numa escala de resposta de tipo Likert com 5 pontos $(1$ - nunca/raramente, 5 - sempre). A aferição do instrumento à amostra estabeleceu uma estrutura fatorial de 10 subescalas (22 itens; e.g., Tento encontrar uma estratégia que me ajude no que tenho de fazer.), são elas: Coping Ativo, Suporte, Expressão de Sentimentos, Reinterpretação Positiva, Humor, Religiosidade, Desinvestimento Comportamental, Negação, Autoculpabilização e Consumo de Substâncias. Esta estrutura demonstrou resultados satisfatórios nos indicadores de ajustamento global $\left[\chi^{2} / \mathrm{gl}=3.25 ; \mathrm{CFI}=0.96\right.$; RMSEA $=0.04$; $p_{R S M E A}=1.000 ;$ SRMR $=.037 ; \lambda>.62$; VEM $\left.>.49 ; \mathrm{CR}>.68\right]$ e nos valores de consistência interna das subescalas [.64 $>\alpha<.86 ; 9$ dimensões têm $\alpha>.70]$.

Os mecanismos de proteção ao nível familiar incluem quer crenças positivas face ao futuro otimismo - partilhadas pelos membros da família do participante, quer as qualidades dos relacionamentos estabelecidos com a mãe, o pai e outra pessoa significativa. Para avaliação do otimismo familiar utilizou-se a versão portuguesa do Life Orientation Test-Revised (Laranjeira, 2008; Scheier \& Carver, 1985). Os seis itens da escala foram reformulados, de modo a permitir aos participantes pronunciarem-se sobre a sua família, e não sobre si mesmos (e.g., Na minha família, em situações difíceis, geralmente espera-se sempre o melhor.). Os itens foram respondidos numa escala de resposta de tipo Likert com 5 pontos ( 1 - discordo totalmente, 5 - concordo totalmente). A versão adaptada para este estudo demostrou um bom ajustamento global, após a exclusão de um item $\left[\chi^{2} / \mathrm{gl}=2.51 ; \mathrm{CFI}=0.99\right.$; RMSEA $=0.02 ; p_{R S M E A}=1.000 ;$ SRMR $\left.=.034 ; \lambda>.62 ; \mathrm{VEM}>.49 ; \mathrm{CR}>.68 ; \alpha=.76\right]$.

Para avaliar a qualidade das relações com a mãe e o pai (ou alternativamente outras/os cuidadoras/es significativos), utilizou-se a versão portuguesa do Network of Relationships Inventory (Furman \& Buhrmester, 1985, 1992; Mendonça \& Fontaine, 2013). Quatro subescalas do instrumento foram selecionadas: satisfação (3 itens; e.g., Sinto-me satisfeito/a com a relação que tenho com ele/a.); intimidade (3 itens; e.g., Falo com ele/a sobre todo o tipo de assuntos.) conflito (3 itens; e.g., Zangamo-nos.) e admiração/valorização percebida (3 itens; e.g., Trata-me com admiração e respeito.). Estes foram respondidos numa escala de resposta de tipo Likert com 5 pontos $(1-$ nunca/raramente, 5 - sempre $)$. As $\mathrm{AFCs}$ da escala, assumindo que se trata de quatro fatores correlacionados, revelaram índices de ajustamento global satisfatórios para cada uma das pessoas consideradas [Mãe: $\chi^{2} / g l=4.90 ; \mathrm{CFI}=0.99$; RMSEA $=0.04 ; p_{R S M E A}=1.000 ;$ SRMR $=.024 ; \lambda>.65 ; \mathrm{VEM}>.55 ; \mathrm{CR}>.78 ; \mathrm{Pai}: \chi^{2} / \mathrm{gl}=4.20 ; \mathrm{CFI}=0.99$; RMSEA $=0.03 ; p_{R S M E A}=1.000 ;$ SRMR $\left.=.018 ; \lambda>0.66 ; \mathrm{VEM}>.58 ; \mathrm{CR}>.81\right]$. Os valores de consistência interna de cada dimensão são bons [ $\alpha>$.78].

\section{Procedimento de análise dos dados}

De forma a explorar quais os mecanismos que predizem a saúde mental e o desajustamento externo, foram testados modelos de regressão em cada um dos grupos em função da etnia e orientação sexual (jovens brancos, jovens negros, jovens heterossexuais e jovens LGB). Considerando que as amostras dos grupos minoritários são de menor tamanho, impedindo a análise de regressão de incluir todas as dimensões em estudo, optou-se por, num primeiro momento, conduzir diversas regressões independentes organizadas em (1) características sociodemográficas, (2) mecanismos de risco, (3) mecanismos individuais e (4) mecanismos familiares. Num segundo momento, foram somente incluídos nos modelos os preditores que se revelaram significativos e com uma magnitude de associação considerada relevante no primeiro momento $(\beta>.150)$ (Ferguson, 2009).

\section{RESULTADOS}

Nesta secção iremos reportar os resultados dos modelos de regressão realizados para a saúde mental e o desajustamento externo, tendo como preditores as dimensões sociodemográficas, os mecanismos de risco e de proteção. Os resultados dos modelos de regressão à saúde mental apresentam-se nas Tabelas 1 e 2 e os relativos ao desajustamento externo nas Tabelas 3 e 4. 


\section{Saúde mental}

0 modelo de regressão elaborado com base nas dimensões que haviam demonstrado predizer a saúde mental de forma significativa e relevante no primeiro momento (Tabela 1) explica, no segundo momento, $39 \%$ da variância da saúde mental dos jovens de minorias raciais (Tabela 2). Neste grupo, maiores níveis de saúde mental são explicados de forma significativa por três variáveis: menor autoculpabilização nas situações stressantes, mais uso de estratégias de coping ativo e maiores níveis de otimismo familiar. 0 poder explicativo destes três mecanismos sobrepõe-se ao efeito negativo direto da perceção de rejeição pessoal e a vitimização baseada em ataques à propriedade, pois estas formas de violência eram preditores significativos da saúde mental, no primeiro momento (Tabela 1), e deixaram de o ser quando inseridos no modelo que inclui os mecanismos de proteção, no segundo momento (Tabela 2).

Tabela 1. Modelos de regressão da saúde mental

\begin{tabular}{|c|c|c|c|c|c|c|c|c|c|}
\hline & \multirow{2}{*}{ Saúde Mental } & \multicolumn{2}{|c|}{ P. Brancos } & \multicolumn{2}{|c|}{ P. Negros } & \multicolumn{2}{|c|}{ P. Heterossexuais } & \multicolumn{2}{|c|}{ P. $L G B$} \\
\hline & & B (EP) & $\boldsymbol{\beta}$ & B (EP) & $\boldsymbol{\beta}$ & B (EP) & $\boldsymbol{\beta}$ & B (EP) & $\boldsymbol{\beta}$ \\
\hline \multirow{4}{*}{ 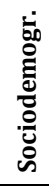 } & Sexo (masc) & $.45(.03)$ & $.27^{* * *}$ & $.49(.11)$ & $.29^{* * *}$ & $.44(.03)$ & $.27^{* * *}$ & $.83(.21)$ & $.40^{* * *}$ \\
\hline & Edu. Par. Secundário & $.05(.04)$ & .03 & $.03(.12)$ & .02 & $.05(.04)$ & .03 & $-.40(.22)$ & $-.21^{+}$ \\
\hline & Edu. Par. Superior & $.08(.04)$ & $.05^{*}$ & $-.07(.15)$ & -.03 & $.06(.04)$ & .03 & $-.05(.23)$ & -.03 \\
\hline & & \multicolumn{2}{|c|}{$\begin{array}{c}F(3,2509)=70.10^{* * *} \\
R^{2}{ }_{\mathrm{a}}=.08\end{array}$} & \multicolumn{2}{|c|}{$\begin{array}{c}F(3,232)=7.10^{* * *} \\
R^{2}{ }_{\mathrm{a}}=.07\end{array}$} & \multicolumn{2}{|c|}{$\begin{array}{c}F(3,2771)=74.06^{* * *} \\
R_{\mathrm{a}}^{2}=.07\end{array}$} & \multicolumn{2}{|c|}{$\begin{array}{c}F(3,81)=7.29^{* * *} \\
R^{2}{ }_{\mathrm{a}}=.19\end{array}$} \\
\hline \multirow{9}{*}{ 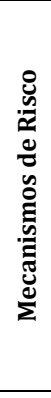 } & Vítima - bullying & $-.14(.03)$ & $-.09 * * *$ & $-.06(.08)$ & -.04 & $-.11(.03)$ & $-.07^{* *}$ & $-.09(.08)$ & -.02 \\
\hline & Vítima - discrimin. & $-.04(.03)$ & -.03 & $-.10(.07)$ & -.09 & $-.04(.03)$ & -.02 & $-.15(.21)$ & -.08 \\
\hline & Tratamento Injusto & $-.11(.02)$ & $-.11^{* * *}$ & $.02(.09)$ & .02 & $-.09(.02)$ & $-.09^{* * *}$ & $-.09(.12)$ & -.10 \\
\hline & Rejeição Pessoal & $-.19(.02)$ & $-.22^{* * *}$ & $-.17(.06)$ & $-.20 * *$ & $-.19(.02)$ & $-.22^{* * *}$ & $-.22(.10)$ & $-.26^{*}$ \\
\hline & Vit. Física & $.13(.03)$ & $.09 * * *$ & $.03(.12)$ & .02 & $.13(.03)$ & $.09^{* * *}$ & $.16(.16)$ & .15 \\
\hline & Vit. Social & $-.12(.02)$ & $-.13^{* * *}$ & $-.14(.08)$ & $-.14^{+}$ & $-.13(.02)$ & $-.13^{* * *}$ & $-.31(.13)$ & $-.37 *$ \\
\hline & Vit. Verbal & $-.06(.02)$ & $-.07 *$ & $.07(.09)$ & .08 & $-.06(.02)$ & $-.08^{* *}$ & $-.08(.13)$ & -.11 \\
\hline & Vit. A. Propriedade & $-.00(.03)$ & -.00 & $-.22(.10)$ & $-.20 *$ & $-.02(.03)$ & -.02 & $.21(.14)$ & .23 \\
\hline & & \multicolumn{2}{|c|}{$\begin{array}{c}F(8,2522)=82.48^{* * *} \\
R_{\mathrm{a}}^{2}=.21\end{array}$} & \multicolumn{2}{|c|}{$\begin{array}{c}F(8,239)=4.56^{* * *} \\
R_{\mathrm{a}}^{2}=.11 \\
\end{array}$} & \multicolumn{2}{|c|}{$\begin{array}{c}F(8,2792)=81.47^{* * *} \\
R_{\mathrm{a}}^{2}=.19\end{array}$} & \multicolumn{2}{|c|}{$\begin{array}{c}F(8,83)=4.49^{* * *} \\
R_{\mathrm{a}}^{2}=.25\end{array}$} \\
\hline \multirow{15}{*}{ 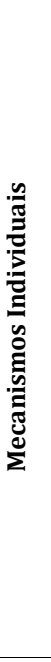 } & P. Ansiosa & $-.64(.03)$ & $-.48^{* * *}$ & $-.58(.10)$ & $-.37^{* * *}$ & $-.62(.03)$ & $-.46^{* * *}$ & $-.89(.15)$ & $-.60^{* * *}$ \\
\hline & T. Perspetiva & $.10(.03)$ & $.08^{* * *}$ & $.05(.09)$ & -.04 & $.11(.02)$ & $.08^{* * *}$ & $.05(.15)$ & .04 \\
\hline & Preoc. Empática & $.01(.03)$ & .01 & $-.12(.09)$ & -.09 & $.02(.03)$ & .01 & $-.02(.15)$ & -.01 \\
\hline & & \multicolumn{2}{|c|}{$\begin{array}{c}F(3,2528)=233.98^{* * *} \\
R^{2}=.22\end{array}$} & \multicolumn{2}{|c|}{$\begin{array}{c}F(3,238)=15.30^{* * *} \\
R^{2}{ }^{2}=.15\end{array}$} & \multicolumn{2}{|c|}{$\begin{array}{c}F(3,2797)=241.84^{* * *} \\
R^{2}{ }_{\mathrm{a}}=.21\end{array}$} & \multicolumn{2}{|c|}{$\begin{array}{c}F(3,82)=14.23^{* * *} \\
R_{\mathrm{a}}^{2}=.33\end{array}$} \\
\hline & C. Ativo & $.07(.02)$ & $.08^{* *}$ & $.22(.07)$ & $.23^{* *}$ & $.08(.02)$ & $.08^{* * *}$ & $.16(.13)$ & .18 \\
\hline & C. Suporte & $-.03(.02)$ & -.03 & $.02(.06)$ & .03 & $-.02(.02)$ & -.02 & $-.12(.10)$ & -.16 \\
\hline & C. Reinterp. Positiva & $.10(.02)$ & $.13^{* * *}$ & $.10(.05)$ & $.13^{+}$ & $.10(.02)$ & $.12^{* * *}$ & $.17(.10)$ & .22 \\
\hline & C. Expr. Sentimentos & $-.00(.02)$ & -.00 & $-.09(.06)$ & $-.11^{+}$ & $-.01(.02)$ & -.01 & $-.09(.09)$ & -.13 \\
\hline & C. Humor & $.13(.01)$ & $.18^{* * *}$ & $.10(.04)$ & $.14^{*}$ & $.13(.01)$ & $.17^{* * *}$ & $.21(.08)$ & $.26^{*}$ \\
\hline & C. Religioso & $-.00(.01)$ & -.00 & $-.03(.04)$ & -.05 & $-.00(.01)$ & -.00 & $.03(.09)$ & .03 \\
\hline & C. Desinvestimento & $-.12(.02)$ & $-.12^{* * *}$ & $-.10(.06)$ & -.10 & $-.12(.02)$ & $-.13^{* * *}$ & $.00(.10)$ & .01 \\
\hline & C. Negação & $-.10(.02)$ & $-.12^{* * *}$ & $-.07(.05)$ & -.09 & $-.11(.02)$ & $-.13^{* * *}$ & $-.04(.09)$ & -.04 \\
\hline & C. Autoculpabilização & $-.24(.01)$ & $-.32^{* * *}$ & $-.26(.05)$ & $-.34^{* * *}$ & $-.24(.01)$ & $-.31^{* * *}$ & $-.28(.08)$ & $-.38^{* *}$ \\
\hline & \multirow[t]{2}{*}{ C. Cons. Substâncias } & $-.09(.02)$ & $-.07^{* * *}$ & $-.06(.08)$ & .04 & $-.06(.02)$ & $-.05^{* *}$ & $-.06(.10)$ & -.06 \\
\hline & & \multicolumn{2}{|c|}{$\begin{array}{c}F(10,2525)=112.27^{* * *} \\
R^{2}{ }_{a}=.31\end{array}$} & \multicolumn{2}{|c|}{$\begin{array}{c}F(10,233)=10.32^{* * *} \\
R^{2}{ }^{2}=.29\end{array}$} & \multicolumn{2}{|c|}{$\begin{array}{c}F(10,2788)=116.45^{* * *} \\
R^{2}{ }_{a}=.29\end{array}$} & $\begin{array}{r}F(10,82 \\
R^{2}{ }_{\mathrm{a}}\end{array}$ & $\begin{array}{l}=5.34^{* * *} \\
35\end{array}$ \\
\hline & Otimismo familiar & $.25(.03)$ & $.21^{* * *}$ & $.30(.09)$ & $.25^{* *}$ & $.25(.02)$ & $.21^{* * *}$ & $.30(.16)$ & $.23^{+}$ \\
\hline & R. Satisf. Mãe & $.21(.03)$ & $.23^{* * *}$ & $.43(.10)$ & $.50^{* * *}$ & $.23(.03)$ & $.25^{* * *}$ & $-.04(.15)$ & -.05 \\
\hline$\stackrel{2}{=}$ & R. Satisf. Pai & $.00(.03)$ & .00 & $-.05(.09)$ & -.07 & $.00(.03)$ & .00 & $-.10(.14)$ & -.13 \\
\hline 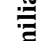 & R. Conf. Mãe & $-.02(.02)$ & -.02 & $.00(.07)$ & .01 & $-.02(.02)$ & -.02 & $-.19(.16)$ & -.19 \\
\hline 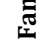 & R. Conf. Pai & $-.10(.02)$ & $-.11^{* * *}$ & $-.09(.06)$ & -.12 & $-.09(.03)$ & $-.10^{* * *}$ & $-.21(.13)$ & $-.23^{+}$ \\
\hline$\overline{\tilde{n}}$ & R. Intim. Mãe & $-.08(.02)$ & $-.12^{* * *}$ & $.03(.06)$ & .04 & $-.08(.02)$ & $-.12^{* * *}$ & $-.14(.12)$ & -.19 \\
\hline. & R. Intim. Pai & $.07(.02)$ & $.10^{* * *}$ & $.04(.07)$ & .06 & $.08(.02)$ & $.11^{* * *}$ & $.07(.14)$ & .08 \\
\hline క్ర్లే & R. Admir. Mãe & $-.02(.03)$ & -.02 & $-.13(.11)$ & -.15 & $-.03(.03)$ & -.03 & $.12(.19)$ & .13 \\
\hline$\sum^{\frac{e}{n}}$ & R. Admir. Pai & $.03(.03)$ & .04 & $.02(.11)$ & .03 & $.04(.03)$ & .05 & $.09(.19)$ & .10 \\
\hline & & $\begin{array}{r}F(9,2364) \\
R_{\mathrm{a}}^{2}\end{array}$ & $\begin{array}{l}=33.44^{* * *} \\
.18\end{array}$ & $\begin{array}{r}F(9,181 \\
R^{2}{ }_{\mathrm{a}}\end{array}$ & $\begin{array}{l}=9.00^{* * *} \\
=.29\end{array}$ & $\begin{array}{r}F(9,2364) \\
R^{2} \mathrm{a}\end{array}$ & $\begin{array}{l}=66.59^{* * *} \\
.19\end{array}$ & $\begin{array}{r}F(9,7 \\
R^{2}\end{array}$ & $\begin{array}{l}=2.14^{*} \\
12\end{array}$ \\
\hline
\end{tabular}

Notas: ${ }^{+} p<.1{ }^{*} p<.05 ;{ }^{* *} p<.01{ }^{* * *} p<.001$ 
Nos jovens de minorias sexuais, o modelo de regressão elaborado explica $61 \%$ da variância da saúde mental dos participantes (Tabela 2). Maior saúde mental é explicada, por ordem de relevância, por: menor vitimização social, menor personalidade ansiosa, ter sofrido ataques à propriedade (variável cujo efeito não era significativo no modelo que considera apenas as diferentes formas de violência), menor autoculpabilização, ser do sexo masculino e mais uso de coping de humor. De forma similar ao que se observou junto dos jovens negros, os mecanismos de proteção considerados neste estudo explicam suficientemente as variações na saúde mental, demonstrando ser mais determinantes que a influência negativa da perceção de rejeição pessoal, sendo que o efeito direto desta forma de violência deixou de ser significativo. No entanto, o mesmo fenómeno não se observa em relação à vitimização social exercida pelos pares, que continua a ser um forte preditor de pior saúde mental na presença dos mecanismos de proteção.

Tabela 2. Preditores da saúde mental

\begin{tabular}{|c|c|c|c|c|c|c|c|c|}
\hline \multirow{2}{*}{ Saúde Mental } & \multicolumn{2}{|c|}{ P. Brancos } & \multicolumn{2}{|c|}{ P. Negros } & \multicolumn{2}{|c|}{ P. Heterossexuais } & \multicolumn{2}{|c|}{ P. $L G B$} \\
\hline & B (EP) & $\beta$ & B (EP) & $\beta$ & B (EP) & $\beta$ & B (EP) & $\beta$ \\
\hline Sexo (masc) & $.09(.02)$ & $.06^{* * *}$ & $.04(.09)$ & .03 & $.17(.03)$ & $.10^{* * *}$ & $.44(.17)$ & $.21^{*}$ \\
\hline Rejeição Pessoal & $-.13(.01)$ & $-.17^{* * *}$ & $-.01(.04)$ & -.02 & $-.12(.02)$ & $-.14^{* * *}$ & $-.08(.08)$ & -.09 \\
\hline Vit. Social & $-.01(.02)$ & -.02 & $-.02(.05)$ & -.03 & $-.05(.02)$ & $-.06^{* *}$ & $-.32(.09)$ & $-38^{* * *}$ \\
\hline Vit. A. Propriedade & $-.00(.02)$ & -.00 & $.07(.06)$ & .08 & $-.03(.02)$ & -.02 & $.22(.09)$ & $.23^{*}$ \\
\hline P. Ansiosa & $-.15(.02)$ & $-.13^{* * *}$ & $-.06(.08)$ & -.05 & $-.31(.02)$ & $-.23^{* * *}$ & $-.41(.13)$ & $-.27^{* *}$ \\
\hline C. Ativo & $.24(.01)$ & $.28^{* * *}$ & $.21(.05)$ & $.29^{* * *}$ & $.10(.02)$ & $.10^{* * *}$ & $.11(.07)$ & .11 \\
\hline C. Humor & $.05(.01)$ & $.08^{* * *}$ & $.04(.03)$ & .06 & $.08(.01)$ & $.11^{* * *}$ & $.14(.06)$ & $.18^{*}$ \\
\hline C. Autoculpabilização & $-.24(.01)$ & $-.37^{* * *}$ & $-.25(.04)$ & $-.40^{* * *}$ & $-.17(.01)$ & $-.22^{* * *}$ & $-.16(.06)$ & $-.21^{*}$ \\
\hline Otimismo familiar & $.13(.02)$ & $.13^{* * *}$ & $.18(.06)$ & $.19^{* *}$ & $.16(.02)$ & $.14^{* * *}$ & $.09(.11)$ & .06 \\
\hline \multirow[t]{2}{*}{ R. Satisf. Mãe } & $.04(.01)$ & $.05^{* *}$ & $.06(.04)$ & .08 & $.11(.02)$ & $.12^{* * *}$ & $.04(.06)$ & .05 \\
\hline & \multicolumn{2}{|c|}{$\begin{array}{c}F(10,2459)=217.16^{* * *} \\
R^{2}{ }_{\mathrm{a}}=.47\end{array}$} & \multicolumn{2}{|c|}{$\begin{array}{c}F(10,210)=14.47^{* * *} \\
R^{2} \mathrm{a}=.39\end{array}$} & \multicolumn{2}{|c|}{$\begin{array}{c}F(10,2692)=195.54^{* * *} \\
R^{2} \mathrm{a}=.42\end{array}$} & \multicolumn{2}{|c|}{$\begin{aligned} F(10,80) & =13.58^{* * *} \\
R^{2} \mathrm{a} & =.61\end{aligned}$} \\
\hline
\end{tabular}

Notas: ${ }^{+} p<.1{ }^{*} p<.05 ;{ }^{* *} p<.01^{* * *} p<.001$

Entre os mecanismos que se revelam significativos, observa-se que a personalidade ansiosa e a autoculpabilização funcionam como mecanismos de vulnerabilidade para a saúde mental. Já o uso do humor nas situações stressantes é o único fator compensador da influência da violência social na saúde mental. $\mathrm{O}$ uso do humor é uma estratégia benéfica também para a saúde mental dos jovens dos grupos maioritários, porém a sua influência é mais pronunciada nos jovens LGB.

Os mecanismos de risco e proteção observados nos grupos minoritários também exercem uma influência significativa na saúde mental dos jovens de grupos maioritários (brancos e heterossexuais). As variáveis consideradas no modelo estão associadas a $42 \%$ e $47 \%$ da variação da saúde mental e níveis mais elevados desta estão associados a: menor autoculpabilização, menor personalidade ansiosa, maior uso de coping ativo, menor rejeição pessoal, maior otimismo familiar, relações mais satisfatórias com a mãe e ser do sexo masculino. Junto dos jovens de grupos maioritários, observa-se que a influência direta da perceção de rejeição pessoal não é anulada pelos mecanismos individuais e familiares.

De forma global, observa-se que a autoculpabilização nos momentos de stress é o preditor com maior influência direta na saúde mental nos jovens heterossexuais, independentemente de se descreverem como brancos ou como negros. Já nos jovens LGB, a dimensão com maior influência direta na saúde mental é a vitimização social exercida pelos pares. Adicionalmente, estima-se que algumas variáveis que predizem de forma significativa a saúde mental dos jovens de grupos maioritários e que manifestam um tamanho de efeito relativamente baixo (como a satisfação na relação com a mãe ou o género) apenas são preditores significativos devido ao tamanho das amostras destes grupos. Assim, valores de regressão padronizados relativamente baixos manifestam significância estatística, enquanto nos grupos minoritários isso não se observa.

\section{Desajustamento externo}

As variáveis incluídas no modelo de regressão delineado com base nas dimensões que haviam demonstrado predizer a saúde mental de forma significativa num primeiro momento (Tabela 3) encontram-se associadas a 19\% da variância do desajustamento externo dos jovens negros num segundo momento (Tabela 4). As variáveis com maior associação ao desajustamento externo manifestado são: o consumo de substâncias como forma de coping, ter sofrido maior vitimização física pelos pares, o desinvestimento comportamental nos momentos de stress e não ter um/a cuidador/a com escolaridade 
de nível superior. Percursos de menor desajustamento externo associam-se ainda a maiores níveis de tratamento injusto no dia a dia.

Tabela 3. Modelos de regressão do desajustamento externo

\begin{tabular}{|c|c|c|c|c|c|c|c|c|c|}
\hline \multirow{2}{*}{\multicolumn{2}{|c|}{ Desajustamento externo }} & \multicolumn{2}{|c|}{ P. Brancos } & \multicolumn{2}{|c|}{ P. Negros } & \multicolumn{2}{|c|}{ P. Heterossexuais } & \multicolumn{2}{|c|}{ P. $L G B$} \\
\hline & & B (EP) & $\boldsymbol{\beta}$ & B (EP) & $\boldsymbol{\beta}$ & B (EP) & $\boldsymbol{\beta}$ & B (EP) & $\boldsymbol{\beta}$ \\
\hline \multirow{4}{*}{ 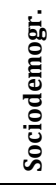 } & Sexo (masc) & $.47(.05)$ & $.18^{* * *}$ & $.33(.17)$ & $.12^{+}$ & $.50(.05)$ & $.19^{* * *}$ & $-.11(.32)$ & -.04 \\
\hline & Edu. Par. Secundário & $-.23(.06)$ & $-.09^{* * *}$ & $.23(.19)$ & .08 & $-.21(.06)$ & $-.08^{* * *}$ & $-.39(.34)$ & -.15 \\
\hline & Edu. Par. Superior & $-.37(.06)$ & $-.13^{* * *}$ & $-.68(.24)$ & $-.19^{* *}$ & $-.41(.06)$ & $-.15^{* * *}$ & $-.28(.35)$ & -.10 \\
\hline & & \multicolumn{2}{|c|}{$\begin{array}{c}F(3,2518)=38.21^{* * *} \\
R^{2}{ }_{\mathrm{a}}=.04\end{array}$} & \multicolumn{2}{|c|}{$\begin{array}{c}F(3,234)=5.57^{* *} \\
R^{2}=.06\end{array}$} & \multicolumn{2}{|c|}{$\begin{aligned} F(3,2782) & =148.70^{* * *} \\
R^{2} & =.05\end{aligned}$} & \multicolumn{2}{|c|}{$\begin{array}{c}F(3,81)=0.49, p=.691 \\
R_{\mathrm{a}}^{2}=-.02\end{array}$} \\
\hline \multirow{9}{*}{ 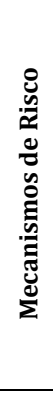 } & Vítima - bullying & $-.01(.06)$ & -.01 & $.16(.10)$ & $.11^{+}$ & $.04(.05)$ & .02 & $-.02(.12)$ & -.01 \\
\hline & Vítima - discrimin. & $.07(.05)$ & .03 & $.17(.12)$ & .09 & $.12(.05)$ & $.05^{*}$ & $.53(.30)$ & $.21^{+}$ \\
\hline & Tratamento Injusto & $.14(.04)$ & $.09^{* *}$ & $-.27(.14)$ & $-.17^{+}$ & $.10(.04)$ & $.07^{* *}$ & $-.09(.18)$ & -.02 \\
\hline & Rejeição Pessoal & $.10(.03)$ & $.07^{* *}$ & $.25(.10)$ & $.19^{*}$ & $.15(.03)$ & $.11^{* * *}$ & $-.23(.15)$ & -.20 \\
\hline & Vit. Física & $.55(.05)$ & $.25^{* * *}$ & $.40(.18)$ & $.17^{*}$ & $.56(.05)$ & $.25^{* * *}$ & $.49(.23)$ & $.33^{*}$ \\
\hline & Vit. Social & $.21(.04)$ & $.14^{* * *}$ & $.03(.13)$ & .02 & $.18(.04)$ & $.12^{* * *}$ & $.62(.19)$ & $.54^{* *}$ \\
\hline & Vit. Verbal & $-.19(.04)$ & $-.15^{* * *}$ & $-.04(.14)$ & -.03 & $-.22(.04)$ & $-.17^{* * *}$ & $-.53(.18)$ & $-.54^{* *}$ \\
\hline & Vit. A. Propriedade & $.02(.05)$ & .09 & $.22(.16)$ & .13 & $.03(.05)$ & .015 & $.02(.20)$ & .01 \\
\hline & & \multicolumn{2}{|c|}{$\begin{array}{c}F(8,2534)=43.94^{* * *} \\
R_{\mathrm{a}}^{2}=.12 \\
\end{array}$} & \multicolumn{2}{|c|}{$\begin{array}{c}F(8,241)=3.61^{* *} \\
R^{2}{ }_{\mathrm{a}}=.08\end{array}$} & \multicolumn{2}{|c|}{$\begin{array}{c}F(8,2804)=47.70^{* * *} \\
R_{\mathrm{a}}^{2}=.12\end{array}$} & \multicolumn{2}{|c|}{$\begin{array}{c}F(8,83)=3.00^{* *} \\
R^{2}{ }_{\mathrm{a}}=.16\end{array}$} \\
\hline \multirow{15}{*}{ 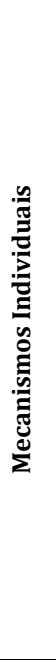 } & P. Ansiosa & $-.14(.05)$ & $-.07 * *$ & $-.21(.17)$ & -.09 & $-.17(.04)$ & $-.08^{* * *}$ & $-.29(.25)$ & -.14 \\
\hline & T. Perspetiva & $-.18(.04)$ & $-.09^{* * *}$ & $-.18(.14)$ & -.09 & $-.21(.04)$ & $-.10^{* * *}$ & $.25(.24)$ & -.13 \\
\hline & Preoc. Empática & $-.04(.05)$ & -.02 & $-.09(.16)$ & -.04 & $-.04(.05)$ & -.02 & $.18(.25)$ & .10 \\
\hline & & \multicolumn{2}{|c|}{$\begin{array}{c}F(3,2533)=13.99^{* * *} \\
R_{\mathrm{a}}^{2}=.02\end{array}$} & \multicolumn{2}{|c|}{$\begin{array}{c}F(3,239)=1.81, p=.147 \\
R_{\mathrm{a}}^{2}=.01\end{array}$} & \multicolumn{2}{|c|}{$\begin{array}{c}F(3,2803)=22.60^{* * *} \\
R_{\mathrm{a}}^{2}=.02\end{array}$} & \multicolumn{2}{|c|}{$\begin{array}{c}F(3,82)=0.96, p=.418 \\
R^{2}{ }_{\mathrm{a}}=-.00\end{array}$} \\
\hline & C. Ativo & $.14(.04)$ & $.09^{* * *}$ & $-.06(.06)$ & -.04 & $.09(.04)$ & $.06^{* * *}$ & $.55(.22)$ & $.44^{*}$ \\
\hline & C. Suporte & $-.07(.03)$ & $-.05^{*}$ & $.03(.05)$ & .03 & $-.07(.03)$ & $-.05^{*}$ & $-.12(.17)$ & -.11 \\
\hline & C. Reinterp. Positiva & $-.15(.03)$ & $-.12^{* * *}$ & $-.04(.05)$ & -.03 & $-.12(.03)$ & $-.09 * * *$ & $-.28(.17)$ & $-.27^{+}$ \\
\hline & C. Expr. Sentimentos & $.06(.03)$ & $.05^{*}$ & $-.09(.05)$ & -.07 & $.05(.03)$ & $.04^{+}$ & $.04(.16)$ & .04 \\
\hline & C. Humor & $.09(.02)$ & $.08^{* * *}$ & $.05(.04)$ & .05 & $.08(.02)$ & $.07^{* * *}$ & $-.07(.14)$ & -.06 \\
\hline & C. Religioso & $-.06(.02)$ & $-.05^{* *}$ & $-.02(.04)$ & -.02 & $-.04(.02)$ & $-.04 *$ & $.07(.15)$ & .06 \\
\hline & C. Desinvestimento & $.08(.03)$ & $.05^{*}$ & $.28(.05)$ & $.19^{* *}$ & $.09(.03)$ & $.06^{* *}$ & $.03(.17)$ & .03 \\
\hline & C. Negação & $.08(.03)$ & $.06^{* *}$ & $.05(.05)$ & .04 & $.10(.03)$ & $.07^{* * *}$ & $-.07(.15)$ & -.06 \\
\hline & C. Autoculpabilização & $-.02(.03)$ & -.02 & $-.10(.05)$ & -.08 & $-.03(.02)$ & $-.03^{* *}$ & $-.13(.14)$ & -.13 \\
\hline & \multirow[t]{2}{*}{ C. Cons. Substâncias } & $.57(.04)$ & $.31^{* * *}$ & $.45(.07)$ & $.20^{* *}$ & $.58(.04)$ & $.30^{* * *}$ & $.16(.17)$ & .11 \\
\hline & & \multicolumn{2}{|c|}{$\begin{array}{c}F(10,2534)=37.52^{* * *} \\
R_{\mathrm{a}}^{2}=.13\end{array}$} & \multicolumn{2}{|c|}{$\begin{array}{c}F(10,234)=2.54^{* *} \\
R^{2}{ }_{a}=.06\end{array}$} & \multicolumn{2}{|c|}{$\begin{array}{c}F(10,2796)=39.67^{* * *} \\
R_{\mathrm{a}}^{2}=.12\end{array}$} & $\begin{array}{r}F(10,82)= \\
R^{2}{ }_{\mathrm{a}}\end{array}$ & $\begin{array}{l}.11, p=.367 \\
.01\end{array}$ \\
\hline & Otimismo familiar & $-.16(.04)$ & $-.09^{* * *}$ & $-.22(.17)$ & -.11 & $-.16(.04)$ & $-.09 * * *$ & $.13(.25)$ & .07 \\
\hline & R. Satisf. Mãe & $.03(.05)$ & .02 & $-.19(.19)$ & -.13 & $.06(.05)$ & .04 & $-.12(.22)$ & -.10 \\
\hline$\stackrel{\circlearrowright}{\leftrightarrows}$ & R. Satisf. Pai & $-.05(.05)$ & -.05 & $.07(.18)$ & .06 & $-.05(.05)$ & -.05 & $.04(.21)$ & .04 \\
\hline 荨 & R. Conf. Mãe & $.12(.04)$ & $.08^{* *}$ & $.01(.13)$ & .01 & $.11(.04)$ & $.08^{* *}$ & $-.11(.24)$ & -.08 \\
\hline 式 & R. Conf. Pai & $.05(.03)$ & .03 & $.14(.12)$ & .11 & $.06(.03)$ & .05 & $.21(.19)$ & .17 \\
\hline $\bar{g}$ & R. Intim. Mãe & $-.04(.03)$ & -.04 & $.04(.12)$ & .04 & $-.05(.03)$ & -.05 & $.06(.17)$ & .06 \\
\hline 气ี & R. Intim. Pai & $.12(.04)$ & $.10^{* *}$ & $.02(.13)$ & .01 & $.12(.03)$ & $.10^{* * *}$ & $-.19(.22)$ & -.16 \\
\hline ฮี & R. Admir. Mãe & $-.12(.06)$ & $-.08^{*}$ & $-.07(.20)$ & -.05 & $-.14(.05)$ & $-.09 *$ & $-.17(.28)$ & -.14 \\
\hline$\sum^{\infty}$ & R. Admir. Pai & $-.11(.05)$ & $-.09 *$ & $.02(.21)$ & .01 & $-.11(.05)$ & $-.09 *$ & $.33(.28)$ & .28 \\
\hline & & $\begin{array}{r}F(9,2375 \\
R^{2} \mathrm{a}\end{array}$ & $\begin{array}{l}=18.69^{* * *} \\
.06\end{array}$ & $\begin{array}{r}F(9,183)= \\
R^{2}{ }_{\mathrm{a}}\end{array}$ & $\begin{array}{l}.19, p=.302 \\
.01\end{array}$ & $\begin{array}{r}F(9,2584) \\
R_{\mathrm{a}}^{2}\end{array}$ & $\begin{array}{l}=20.33^{* * *} \\
.06\end{array}$ & $\begin{array}{r}F(9,75)= \\
R^{2} \mathrm{a}\end{array}$ & $\begin{array}{l}39, p=.936 \\
.08\end{array}$ \\
\hline
\end{tabular}

Notas: ${ }^{+} p<.1 ;^{*} p<.05 ;{ }^{* *} p<.01{ }^{* * *} p<.001$

Nos jovens LGB, os mecanismos de risco e de proteção avaliados estão associados a $26 \%$ da variação do desajustamento social e/ou escolar. Maior desajustamento externo está associado a: menores níveis de vitimização verbal, maior vitimização social e física exercida pelos pares, maior uso de coping ativo e ter sido vítima de discriminação. Observa-se ainda uma tendência para o consumo de substâncias enquanto estratégia de coping se associar positivamente a um percurso socialmente desajustado.

Nos jovens de grupos maioritários (brancos e heterossexuais), observa-se que as variáveis consideradas no modelo estão associadas a $23 \%$ da variação do desajustamento externo. Este indicador de má-adaptação encontra-se associado a: maior consumo de substâncias como forma de lidar com o stress, ter sofrido maior vitimização física e social por parte de colegas, ser do sexo masculino, não ter 
um/a cuidador/a com escolaridade de nível superior (de forma similar aos jovens negros) e ter sofrido menor vitimização verbal por parte dos pares (de forma similar ao observado nos jovens LGB).

Tabela 4. Preditores do desajustamento externo

\begin{tabular}{|c|c|c|c|c|c|c|c|c|}
\hline \multirow{2}{*}{ Desajustamento externo } & \multicolumn{2}{|c|}{ P. Brancos } & \multicolumn{2}{|c|}{ P. Negros } & \multicolumn{2}{|c|}{ P. Heterossexuais } & \multicolumn{2}{|c|}{ P. $L G B$} \\
\hline & B (EP) & $\boldsymbol{\beta}$ & B (EP) & $\boldsymbol{\beta}$ & B (EP) & $\boldsymbol{\beta}$ & B (EP) & $\boldsymbol{\beta}$ \\
\hline Sexo (masc) & $.41(.05)$ & $.16^{* * *}$ & $.32(.18)$ & $.12^{+}$ & $.42(.05)$ & $.16^{* * *}$ & $-.37(.32)$ & -.13 \\
\hline Edu. Par. Superior & $-.24(.05)$ & $-.09 * * *$ & $-.54(.23)$ & $-.15^{*}$ & $-.30(.05)$ & $-.11^{* * *}$ & $-.31(.28)$ & -.11 \\
\hline Vítima - discrimin. & $.10(.05)$ & $.04^{*}$ & $.12(.11)$ & .07 & $.13(.04)$ & $.05^{* *}$ & $.66(.30)$ & $.26^{*}$ \\
\hline Tratamento Injusto & $.10(.04)$ & $.07^{* *}$ & $-.28(.14)$ & $-.18^{*}$ & $.07(.04)$ & $.05^{+}$ & $.01(.18)$ & .01 \\
\hline Rejeição Pessoal & $.05(.03)$ & .04 & $.18(.10)$ & $.14^{+}$ & $.09(.03)$ & $.07^{* *}$ & $-.21(.15)$ & -.18 \\
\hline Vit. Física & $.41(.05)$ & $.19^{* * *}$ & $.43(.18)$ & $.19^{*}$ & $.41(.05)$ & $.19^{* * *}$ & $.62(.22)$ & $.41^{* *}$ \\
\hline Vit. Social & $.24(.04)$ & $.16^{* * *}$ & $.14(.12)$ & .09 & $.21(.03)$ & $.14^{* * *}$ & $.61(.19)$ & $.53^{* *}$ \\
\hline Vit. Verbal & $-.18(.04)$ & $-.14^{* * *}$ & $-.03(.13)$ & -.02 & $-.18(.03)$ & $-.14^{* * *}$ & $-.68(.18)$ & $-.69 * * *$ \\
\hline C. Ativo & $.11(.03)$ & $.07^{* *}$ & $-.10(.11)$ & -.07 & $.07(.03)$ & $.05^{*}$ & $.36(.18)$ & $.28^{*}$ \\
\hline C. Reinterp. Positiva & $-.08(.03)$ & $-.07^{* *}$ & $-.02(.09)$ & -.02 & $-.06(.02)$ & $-.05^{*}$ & $-.20(.14)$ & -.20 \\
\hline C. Desinvestimento & $.04(.03)$ & .03 & $.23(.10)$ & $.16^{*}$ & $.04(.03)$ & .03 & $-.12(.13)$ & -.10 \\
\hline \multirow[t]{2}{*}{ C. Cons. Substâncias } & $.51(.04)$ & $.27^{* * *}$ & $.61(.17)$ & $.23^{* * *}$ & $.53(.03)$ & $.28^{* * *}$ & $.24(.15)$ & $.18^{+}$ \\
\hline & \multicolumn{2}{|c|}{$\begin{array}{c}\mathrm{F}(12,2499)=61.93^{* * *} \\
R_{\mathrm{a}}^{2}=.23\end{array}$} & \multicolumn{2}{|c|}{$\begin{array}{c}\mathrm{F}(12,226)=5.38^{* * *} \\
R_{\mathrm{a}}^{2}=.19\end{array}$} & \multicolumn{2}{|c|}{$\begin{array}{c}\mathrm{F}(12,2753)=68.19^{* * *} \\
R_{\mathrm{a}}^{2}=.23\end{array}$} & \multicolumn{2}{|c|}{$\begin{array}{c}\mathrm{F}(12,80)=3.33^{* *} \\
R^{2}{ }_{\mathrm{a}}=.26\end{array}$} \\
\hline
\end{tabular}

De forma global, os resultados demonstram que, com exceção do nível de escolaridade parental, as dimensões da qualidade do ambiente familiar não exercem influência no desajustamento externo. Adicionalmente, observa-se que junto dos jovens heterossexuais, independentemente de se descreverem como brancos ou como negros, o desajustamento externo encontra-se mais fortemente associado ao consumo de substâncias enquanto estratégia de coping, enquanto nos jovens LGB está mais associado à violência social.

\section{DISCUSSÃo}

Partindo de uma perspetiva socioecológica da resiliência, que valoriza o contexto específico de cada adversidade, dos mecanismos que poderão exercer funções de proteção e daqueles que podem ser indicadores de ajustamento, procurou-se, ao longo deste trabalho, estudar especificamente o risco psicossocial associado à violência social, nomeadamente a vitimização por pares e a perceção de discriminação, assim como os mecanismos de proteção compensatórios perante estas adversidades. Tendo como foco a discriminação, uma forma de violência social especialmente pervasiva, procurou-se também, ao longo do estudo, estudar os mecanismos que favorecem um ajustamento psicológico de jovens de grupos discriminados, nomeadamente jovens negros e de jovens LGB, comparando-os com jovens brancos e heterossexuais, respetivamente.

\section{Associações da vitimização por pares e da discriminação}

Os resultados das análises de regressão revelam que diversas formas de violência social estão associadas à saúde mental e ao desajustamento externo. Estes resultados reproduzem o que já havia sido observado em diversos estudos sobre a vitimização entre pares e sobre a discriminação (e.g., Hawker \& Boulton, 2000; Russell et al., 2012; Schmitt et al., 2014). Adicionalmente, os resultados revelam que a identificação racial e a orientação sexual moderam o efeito nocivo das várias formas de violência social operacionalizadas no estudo, pelo que a discussão será apresentada tendo em consideração as diferenças entre grupos nos padrões de associação entre as diferentes variáveis. De forma global, contudo, pode observar-se que os resultados sugerem uma manifestação de perturbação diferenciada em função do tipo de agressão sofrida independentemente do grupo de pertença: a perceção de rejeição pessoal associa-se mais fortemente à saúde mental, enquanto a vitimização física está mais associada ao desajustamento social.

\section{A saúde mental e a violência social}

Os resultados revelam que junto dos jovens heterossexuais e negros, o principal preditor de menor saúde mental é a perceção de rejeição pessoal (que traduz uma perceção de desvalorização do próprio pelos outros) (ver Tabela 1 e 2). Já a pior saúde mental dos jovens LGB está mais fortemente associada a ter sofrido vitimização social pelos pares (e.g., exclusão social). Estudos anteriores já haviam demostrado que são as agressões verbais e relacionais, e não a agressão física, que potenciam um mau-ajustamento 
interno (Hawker \& Boulton, 2001). Este efeito poderá dever-se ao facto destas formas de agressão afetarem principalmente os recursos sociais (por oposição aos recursos físicos), que são os mais valorizados na nossa sociedade e com mais implicações no sentido de pertença das crianças e jovens. Interessantemente, os resultados revelam ainda que o preditor mais forte da saúde mental dos jovens brancos e heterossexuais é o constructo de violência mais subtil que foi operacionalizado, a rejeição pessoal. Este fenómeno pode ser explicado pelo sentido de impotência criado pela desvalorização subtil, associado à dificuldade em identificar, e denunciar, como agressão estes comportamentos de violência encoberta. Um estudo no domínio da vitimização por pares revelou que nas situações de agressão aberta, as vítimas são mais capazes de interpretar as intenções do agressor, reagindo assim de forma mais diferenciada a cada tipo de agressão; enquanto em situações de violência indireta, mais subtil, poderão ter mais dificuldades em perceber a intenção e denunciar o ato como sendo uma agressão (Ortega, Elipe, Mora-Merchán, Calmaestra, \& Vega, 2009). Assim, formas de violência mais camufladas (como a rejeição pessoal e a vitimização social) poderão levar a uma menor agência perante o preconceito e perante as situações negativas vividas, o que por sua vez pode contribuir para menor saúde mental (Foster, 2009).

Um resultado inesperado sugere que, junto dos jovens LGB, ter sofrido mais ataques à propriedade está associado a maiores níveis de saúde mental. No entanto, esta forma de vitimização, não se relacionou de forma significativa no modelo exclusivo dos mecanismos de risco. Assim, o caráter inconstante deste resultado e a sua incoerência com o esperado teoricamente e com o que foi observado nos participantes do outro grupo minoritário em estudo torna difícil a sua interpretação.

De sublinhar que apenas no caso específico dos jovens negros, as influências diretas da perceção de rejeição pessoal, da vitimização social e dos ataques à propriedade na saúde mental parecem ser contidas pelos mecanismos de proteção em estudo. Contudo, importa salientar que o processo subjacente a este efeito de amortização da influência direta das várias formas de violência é desconhecido e que estudos futuros deverão explorar este fenómeno. Nos jovens brancos, heterossexuais ou LGB, não se observou uma anulação do efeito direto das várias formas de violência social na saúde mental.

\section{O desajustamento externo e a violência social}

Em todos os grupos em estudo, o desajustamento externo encontra-se associado à vitimização física. A direção das influências entre estas variáveis não pode ser esclarecida no âmbito deste estudo com design transversal. É possível que jovens com pior performance social tenham sido mais rejeitados pelos pares, mas também que a exclusão promova condutas socialmente desajustadas. A relação de causalidade será impossível de estabelecer no presente estudo, de natureza transversal. Uma revisão de estudos longitudinais sobre comportamentos externalizados e vitimização entre pares constatou que as influências ocorrem em ambas as direções, contudo observou-se que o efeito da violência nos comportamentos externalizados é mais forte que no sentido inverso (Reijntjes et al., 2011). Adicionalmente, dado que um dos itens de avaliação de desajustamento externo incluía a possibilidade de ter-se agredido alguém, esta associação poderá também configurar situações de agressão reativa relativamente ao próprio agressor. Não está, assim, posta de lado a hipótese de que uma parte das vítimas se torne agressora, como o salientam estudos no domínio do bullying. Em Portugal, estima-se que entre $6 \%$ a $24 \%$ dos adolescentes corresponda a este perfil de vitimização e agressão simultânea (Carvalhosa, Moleiro, \& Sales, 2009).

Para além da vitimização física pelos pares, observa-se que junto dos jovens LGB, a vitimização social e ter-se sido alvo de discriminação está fortemente associada ao desajustamento externo. Por um lado, este resultado indica que estes jovens parecem estar em maior risco para a discriminação e exclusão social, sendo mais penalizados, quando não cumprem as tarefas socialmente valorizadas (e.g., sucesso académico). Por outro lado, pode também indicar que na presença de exclusão social ou atos de discriminação, os jovens LGB reagem de forma mais exteriorizada (e.g., consumo de substâncias, agressividade, insucesso escolar) do que os jovens heterossexuais (brancos ou negros). Estas formas de violência poderão assim interagir com outros fatores relacionados com a orientação sexual minoritária (e.g., a não aceitação parental ou a homofobia internalizada) para prejudicar o ajustamento psicossocial destes jovens (Fedewa \& Ahn, 2011; Eisenberg \& Resnick, 2006; Hatzenbuehler, 2009; Russell et al., 2012; Ryan et al., 2010; Saewyc, 2011).

De forma inesperada, observou-se que o desajustamento externo apresenta uma associação negativa com o tratamento injusto (junto de jovens negros) e a vitimização verbal (junto de jovens de grupos não discriminados e de minorias sexuais). Recordamos que ambas as subescalas incluem itens de insulto verbal, pelo que estas associações possivelmente expressam uma relação negativa entre ser alvo de agressões verbais e o desajustamento externo. A natureza destas associações precisa de ser esclarecida em função dos níveis de violência sofrida e dos níveis de desajustamento externo. Por um lado, estas associações indicam que os jovens negros que apresentam percursos com maior 
desajustamento externo (e.g., insucesso escolar) são menos insultados e provocados no seu dia a dia; de forma similar, os jovens brancos e LGB com maior desajustamento externo demostram ter sofrido ao longo da vida menos atos de agressão verbal e situações de ridicularização. Nestas situações, pode-se prever que sejam os jovens mais velhos (devido à retenção escolar) que se encontram de alguma forma mais protegidos destas formas de violência. Se for o caso, este resultado é congruente com as observações que a popularidade está associada a atos antissociais (Smetana, Campione-Barr, \& Metzger, 2006) e ao consumo de substâncias (Diego, Field, \& Sanders, 2003), e que os adolescentes em situações de risco desenvolvem estratégias para percecionarem controlo dentro do seu grupo de pares, o que pode levar a um aumento de consumo de substâncias psicoativas de forma a promover o estatuto social (Ungar, 2000). Por outro lado, pode indicar que são os jovens com melhor ajustamento externo, ou seja, os jovens com bom desempenho escolar e comportamental, que são mais frequentemente alvo de insultos verbais. A suportar esta hipótese, num estudo português, observou-se que os alunos com maior desempenho académico afirmaram ter sido mais gozados devido à sua aparência do que os alunos com pior desempenho (Quental \& Veiga, 2010).

\section{Mecanismos de proteção do ajustamento em situações de violência social}

\section{Características sociodemográficas}

No que concerne à influência dos fatores sociodemográficos, os resultados revelam que o nível de escolaridade das figuras parentais (indicador proximal do nível socioeconómico da família) não está associado de forma revelante à saúde mental. Contudo, este indicador está associado de forma significativa ao desajustamento externo dos adolescentes. Ter pelo menos um progenitor com o nível de educação superior está associado a um percurso mais ajustado, com menor insucesso escolar e condutas sociais desadequadas. Estudos portugueses já haviam demonstrado que os jovens de nível socioeconómico baixo, para além de poderem ser mais vítimas de agressão pelos pares, também manifestam maior consumo de substâncias, pior desempenho académico, piores níveis de saúde e menor qualidade de vida em geral (Gaspar, Matos, Ribeiro, \& Leal, 2010; Matos et al., 2004; Pereira, Mendonça, Neto, Valente, \& Smith, 2004). O presente resultado converge, por conseguinte, com o que tem sido amplamente documentado, mais precisamente a influência protetora do estatuto socioecónomico médio ou elevado na competência escolar e social (ajustamento externo), assim como o risco da pobreza para os consumos de substâncias, comportamentos agressivos e atividade ilegais (Coimbra, 2008; Fergus \& Zimmerman, 2005; Masten, 2001, Rutter, 2001). Estudos no âmbito da resiliência têm sustentado que as figuras parentais da classe média e alta fornecem modelos de sucesso e proporcionam oportunidades para o desenvolvimento de perceções de mestria e autoeficácia (e.g., através da participação em atividades extracurriculares), o que, por sua vez, vai estar associado à competência académica e social e ao capital cultural (Abelev, 2009; Coimbra, 2008; Masten \& Reed, 2002). A pobreza tem sido amplamente reconhecida como um mecanismo de risco para o ajustamento, sobretudo porque ao baixo capital económico e social estão associadas diversas adversidades cumulativas (e.g., residir em zonas com fácil acesso a drogas) (Coimbra, 2008; Fergus \& Zimmerman, 2005; Gaspar, Gonçalves, Ramos, \& Matos, 2006; Rutter, 2001). Assim, não é surpreendente que ter pelo menos um dos progenitores com educação de nível superior seja um mecanismo promotor de melhor ajustamento externo. Contudo, este efeito protetor do nível educacional parental não se observa nos jovens LGB, o que indica que o (des)ajustamento destes é mais influenciado por experiências pessoais e pelos mecanismos que utilizam para lidar com eles, do que pelas oportunidades, ou falta delas, proporcionadas pelo contexto do sistema familiar.

0 género revelou também estar associado de forma revelante aos níveis de saúde mental e de ajustamento externo. Foi observado que ser do sexo masculino está associado a maior saúde mental, ao mesmo tempo que também está associado a trajetórias com maior desajustamento externo. De forma global, estes resultados vão de encontro ao que tem sido reportado na literatura, nomeadamente que durante a adolescência as raparigas manifestam maior risco de perturbações internalizadas (como depressão e ansiedade), enquanto nos rapazes o maior risco está associado a externalização (condutas antissociais e consumo de substâncias) (Graham \& Rutter, 1973; Neto, 2010b; Nolen-Hoeksema \& Girgus, 1994; Rose \& Rudolph, 2006; Rutter, Caspi, \& Moffitt, 2003; Rutter, 2005).

\section{Mecanismos individuais}

Ao nível dos mecanismos individuais, a personalidade ansiosa é um preditor significativo de menor saúde mental, resultados que se coadunam com outros em que foi observado que as pessoas resilientes face a diversos tipos de adversidade, em comparação com aqueles que manifestam pior ajustamento, demonstram menor emotividade negativa (Masten, Hubband, Gest, Tellegen, Garmezy, \& Ramirez, 1999) 
e adultos resilientes com um passado de maus-tratos infantis manifestam menor neuroticismo (Collishaw, Pickles, Messer, Rutter, Sheaner, \& Maughan, 2007). Contudo, a personalidade ansiosa não revelou estar associada ao (des)ajustamento externo. Estes resultados sugerem que pessoas com personalidade ansiosa poderão estar mais sensíveis, psicologicamente mais reativas, aos efeitos da violência social, potenciando os comportamentos internalizados, mas não os externalizados. A personalidade ansiosa parece, assim, funcionar como um elemento de sensibilidade perante o risco associado à violência (Rutter, 2001; Ungar, 2013). Por sua vez, as competências de empatia não mostraram estar associadas de forma relevante e significativa à saúde mental, nem ao desajustamento externo. Em outros estudos, a amabilidade (Shiner \& Masten, 2012) e as competências sociais (Vassallo et al., 2014) desempenharam uma função promotora do ajustamento positivo, o que motivou a nossa exploração desta associação. Todavia, os resultados não confirmaram a nossa hipótese.

No que concerne às estratégias de coping, observa-se que a influência do uso de estratégias de coping negativas (nomeadamente a autoculpabilização e o desinvestimento comportamental) é superior ao efeito daquelas mais positivas (coping ativo e de humor). Resultados similares já haviam sido observados num outro estudo com mulheres lésbicas e bissexuais (Kaysen, Kulesza, Balsam, Rhew, Blayney, Lehavot, \& Hughes, 2014). Abordando primeiramente o mecanismo mais influente na saúde mental, a autoculpabilização, estudos prévios já tinham revelado o seu efeito nocivo, potenciando a depressão, ansiedade, solidão e menor autoestima (Graham \& Juvonen, 1998; Kaysen et al., 2014). Deste modo, os resultados sugerem que a autoculpabilização é um mecanismo de vulnerabilidade (Luthar et al., 2000). Adicionalmente, alguns estudos demostram que a vitimização por pares contribui para um aumento da autoculpabilização (Catterson \& Hunter, 2010; Schacter, White, Chang, \& Juvonen, 2015), pelo que é possível que a autoculpabilização seja mediadora dos efeitos da violência social na saúde mental, porém, apenas estudos futuros poderão esclarecer esta hipótese.

Em segundo lugar, destacam-se as associações positivas do desajustamento externo com o desinvestimento comportamental e o consumo de substâncias. A associação do desinvestimento nos projetos pessoais em situação de stress com o desajustamento externo ao longo da vida só é significativa, relevante (em termos de magnitude) e constante (não dependente de outras variáveis) junto dos jovens negros que, como observado em Freitas (2016), com maior probabilidade, vivem situações socioeconómicas desfavorecidas e que são alvo frequente de discriminação. Assim, estes resultados sugerem que as experiências de desajustamento potenciam um certo desânimo, instalando-se um padrão de esgotamento e exaustão perante a adversidade (Masten \& Tellegen, 2012). Em Portugal, já haviam sido reportados elevados níveis de tristeza e desesperança em imigrantes que residem em condições de pobreza (Matos, Simões, \& Gaspar, 2009). As experiências de fracasso social ou académico têm um impacto mais negativo junto destes jovens, provavelmente porque vivem em condições socioeconómicas mais precárias, com mais barreiras sociais, que reduzem substancialmente as suas oportunidades para o sucesso e o desenvolvimento de uma perceção de autoeficácia positiva (Bandura, 2001; Coimbra, 2008; Masten \& Wright, 2010; Rutter, 2001).

Por sua vez, o consumo de substâncias encontra-se associado de forma constante a trajetórias mais desajustadas a nível externo, não só junto dos jovens negros, mas também de jovens de grupos maioritários. Sendo que um dos acontecimentos de vida indicador de desajustamento externo é a dependência de substâncias (álcool, tabaco, outras) esta associação era expectável. Observou-se ainda que nos jovens LGB o consumo de substâncias não está associado de forma significativa ao desajustamento externo, o que sugere um impacto diferencial destes consumos de acordo com o grupo de pertença. Estudos junto dos jovens LGB têm reportado uma associação entre o maior consumo de substâncias junto dos jovens LGB e a violência experienciada (Fedewa \& Ahn, 2011). Já os estudos sobre o consumo de drogas nos jovens de grupos maioritários são muitas vezes atribuídos a uma associação a pares com trajetórias de vida mais desviantes (Ungar, 2000), pelo que junto dos jovens heterossexuais há uma interação dinâmica que se reforça mutuamente entre o desajustamento externo e o consumo de drogas como forma de coping.

Um terceiro ponto digno de atenção está relacionado com as estratégias de coping ativo e de planeamento. No grupo de jovens heterossexuais, observa-se que o coping ativo contribui, conforme o que era previsto, para uma maior saúde mental destes (Hemphill et al., 2014; Herman-Stahl \& Petersen, 1996). Já nos jovens LGB o uso do humor é a estratégia de coping mais influente na saúde mental. Esta influência diferencial em função da orientação sexual poderá dever-se às adversidades específicas enfrentadas por jovens LGB. Estes têm de lidar com o elevado preconceito homofóbico existente nos contextos escolares (António et al., 2012; Rodrigues, Brás, Cunha, Petiz, \& Nogueira, 2015), mas também a gestão da revelação da sua orientação sexual (coming-out). Na interação destas especificidades, o uso de humor parece assim ser a estratégia que permite aos jovens gerir situações stressantes e contribuir para uma maior saúde mental. Observou-se ainda que nos jovens LGB ter um percurso com maior 
desajustamento externo estava associado a maior coping ativo. Este resultado sugere que os jovens LGB que viveram situações de fracasso académico e social redobram o seu esforço para lidar com as situações através de estratégias que visam diminuir o problema (em vez de o evitar). Este esforço desenvolvido pode mesmo ser reflexo de uma trajetória de desafio (Coimbra \& Fontaine, 2015; Masten \& Wright, 2010). Nestas trajetórias observa-se que ter de lidar com situações stressantes, como é o caso do insucesso escolar, poderá favorecer o fortalecimento de estratégias e recursos que se revelam protetoras a longo prazo.

\section{Mecanismos familiares}

O resultado das regressões aos indicadores de ajustamento revela a importância de alguns mecanismos familiares para o ajustamento positivo. 0 otimismo familiar revela-se um fator promotor da saúde mental de jovens brancos, negros e/ou heterossexuais. Deste modo, o otimismo familiar revela ser um forte promotor do ajustamento interno em situações de adversidade, à semelhança do que tem sido reportado noutros estudos para o otimismo individual (e.g., Herman-Stahl \& Petersen, 1996). Estudos anteriores sugerem que, quando o stress é incontrolável, o otimismo associa-se ao uso de estratégias focadas no processamento das emoções, já quando o elemento stressante é controlável, o otimismo está mais fortemente associado ao uso estratégias focadas no problema (Carver \& Connor-Smith, 2010; Carver \& Scheier, 2002). A flexibilidade é também uma característica das famílias resilientes, que se vão adaptando com sucesso a situações de crise (Walsh, 2002, 2003). Os jovens do presente estudo que manifestam melhor ajustamento poderão assim ter beneficiado de modelos que lhes transmitem esperança no futuro e, como tal, os incentivam a desenvolver os esforços adequados quando têm de lidar com situações adversas. Este efeito não parece observar-se nos jovens LGB. 0 otimismo familiar já havia revelado ser preditor da saúde mental nos jovens LGB (Freitas, D'Augelli, Coimbra, \& Fontaine, 2016), porém outros mecanismos, nomeadamente os de violência social e individuais são mais determinantes na saúde mental destes jovens.

No que concerne às qualidades positivas das relações com os pais, observou-se que nos jovens de grupos maioritários, apenas a satisfação na relação com a mãe é promotora da saúde mental. A genderização das interações familiares pode ser uma hipótese explicativa para estas associações diferenciais. Estudos revelam que os adolescentes passam mais tempo com as mães e, com maior probabilidade, partilham assuntos emocionais com estas (Laursen \& Collins, 2009; Smetana et al., 2006), pelo que a satisfação com a relação que estabelecem com esta figura assume também um maior valor preditivo do ajustamento da saúde mental. Estes resultados vão de encontro ao observado no estudo de Herman-Stahl e Petersen (1996), no qual os adolescentes resilientes perante diversos acontecimentos de vida negativos tinham melhores relacionamentos com a família.

De forma surpreendente, os níveis de intimidade com ambas as figuras parentais não revelaram ser preditores do ajustamento. Considerando que as relações filiais em que há intimidade (que implica comunicação e confiança) devem ser globalmente positivas, isto coloca em questão a hipótese dos jovens não estarem atentos ao apoio providenciado pelos pais, mas também poderá dever-se à preferência, pela parte dos adolescentes, do estabelecimento de relações de intimidade junto dos pares (Furman \& Buhrmester, 1992; Lempers \& Clark-Lempers, 1992).

\section{Limitações}

As principais limitações do presente estudo incluem o uso de instrumentos de autorrelato e a recolha de informações apenas junto do participante. A recolha de informações junto da família sobre as relações filiais, assim como junto dos professores e colegas relativamente à vitimização entre pares, iria permitir uma avaliação mais ampla e triangulada do fenómeno em estudo. Uma outra grande limitação do estudo é a sua natureza transversal, não nos permitindo inferir sobre processos de causalidade. Ainda, as medidas de autorrelato transversais também não são todas específicas à situação de violência social, como é o caso das estratégias de coping ou das relações familiares, pelo que apenas a realização de um estudo longitudinal e com medidas mais específicas poderia responder de forma mais rigorosa a algumas das questões em estudo. A não realização de análises moderadoras também não nos permite distinguir entre os mecanismos que apenas compensam os efeitos nocivos da violência social e os que anulam esse efeito, pelo que futuros estudos deverão centrar-se na análise de mecanismos de proteção estabilizadores (Luthar et al., 2000).

\section{Implicações para a intervenção}

Com base nos resultados aqui apresentados, algumas implicações para a intervenção podem ser delineadas. Primeiramente, os resultados das associações entre as diferentes medidas de violência social e a saúde mental sublinharam o impacto negativo das agressões subtis - como ser tratado como inferior 
ou ser excluído das atividades sociais - mais do que qualquer outra forma de violência (ver Tabela 1). Este resultado tem implicações importantes para os agentes educativos, demonstrando que estes não deverão limitar-se a intervir em situações de agressão aberta. A identificação e intervenção nas situações de violência subtil podem constituir um desafio para os educadores, visto que os comportamentos de exclusão entre pares e de ridicularização de alguém não são necessariamente percebidos como bullying (Boulton, 1997). Não obstante, o seu impacto no ajustamento interno revela que os esforços de prevenção devem também ser canalizados para as formas de violência subtis e indiretas.

Os resultados observados confirmam a nossa hipótese global que os mecanismos de proteção desempenham uma função importante e diferenciadora do ajustamento (interno e externo) dos jovens que sofreram alguma forma de violência social. Observa-se que são sobretudo os mecanismos de proteção individuais que mais influenciam os níveis de saúde mental e o desajustamento externo. Desta forma, quando os jovens estão sujeitos a níveis moderados de violência social, para além dos esforços desenvolvidos para cessar essa violência, é importante que sejam desenvolvidas ações no sentido de melhorar as estratégias de coping e estilos de interpretação do meio de modo a promover um ajustamento interno e externo positivo.

Por fim, observou-se que os mecanismos de risco e de proteção que exercem uma influência mais significativa nos indicadores do ajustamento são específicos para cada grupo social, pelo que se destaca a natureza socioecológica do processo de resiliência (Ungar, 2013). Deste modo, para uma correta avaliação do risco e dos recursos a potenciar, revela-se necessária a consideração das interseções entre os vários mecanismos de risco e de proteção de acordo com a especificidade de cada contexto.

A este nível, gostaríamos de destacar que as perspetivas sobre o bullying, mesmo as socioecológicas, têm-se focado na desigualdade de poder entre o agressor e a vítima, e conceptualizado esta diferença sobretudo em termos de força física, popularidade e pelo facto de a agressão ocorrer em grupo (e.g., Hunter, Boyle, \& Warden, 2007). No entanto, os preconceitos sustentam também desigualdades de poder (Krieger, 1999), pelo que as perspetivas socioecológicas do bullying deverão incluir não apenas os processos proximais (comunitários, clima escolar) (Swearer, Espelage, Vaillancourt, \& Hymel, 2010; Swearer \& Hymel, 2015), mas também os preconceitos e a desigualdade social como influências do macrossistema. Alguns dos processos pessoais e grupais que têm sido identificados em situações de bullying - como o distanciamento moral, a culpabilização e infra-humanização da vítima estão também presentes na discriminação (Almeida, Correia, \& Marinho, 2010; Bandura, 2002; CostaLopes, Vala, Pereira, \& Aguiar, 2008; Gato, Carneiro, \& Fontaine, 2011). Neste sentido, as intervenções na vitimização entre pares deverão ser desenvolvidas junto dos diversos elementos, não só do contexto educativo, mas também da sociedade, abordando elementos relativos ao estatuto, desigualdade e privilégio social (Krieger, 1999) e com ações com vista a diminuir os preconceitos (Dessel, 2010).

\section{REFERÊNCIAS}

Abelev, M. S. (2009). Advancing out of poverty: Social class worldview and its relation to resilience. Journal of Adolescent Research, 24, 114-141. https://doi.org/10.1177/0743558408328441

Almeida, A., Correia, I., \& Marinho, S. (2010). Moral disengagement, normative beliefs of peer group, and attitudes regarding roles in bullying. Journal of School Violence, 9(1), 23-36. https://doi.org/10.1080/15388220903185639

António, R., Pinto, T., Pereira, C., Farcas, D., \& Moleiro, C. (2012). Bullying homofóbico no contexto escolar em Portugal. Psicologia, 26(1), 17-32.

APA Task Force on Resilience and Strength in Black Children and Adolescents. (2008). Resilience in African American children and adolescents: A vision for optimal development. Washington, DC: American Psychological Association. Disponível em: https://www.apa.org/pi/families/resources/resiliencerpt.pdf

Averdijk, M., Eisner, M., \& Ribeaud, D. (2014). Do social relationships protect victimized children against internalizing problems? Journal of School Violence, 13(1), 80-99. https://doi.org/10.1080/15388220.2013.842175

Bandura, A. (2001). Social cognitive theory: An agentic perspective. Annual Review of Psychology, 52, 126. https://doi.org/10.1146/annurev.psych.52.1.1

Bandura, A. (2002). Selective moral disengagement in the exercise of moral agency. Journal of Moral Education, 31(2), 101-119. https://doi.org/10.1080/0305724022014322

Boulton, M. J. (1997). Teachers' views on bullying: definitions, attitudes and ability to cope. British Journal of Educational Psychology, 67(2), 223-233. https://doi.org/10.1111/j.2044-8279.1997.tb01239.x

Bowes, L., Maughan, B., Caspi, A., Moffitt, T. E., \& Arseneault, L. (2010). Families promote emotional and behavioural resilience to bullying: evidence of an environmental effect. Journal of Child Osychology and Psychiatry, 51(7), 809-817. https://doi.org/10.1111/j.1469-7610.2010.02216.x 
Carrera-Fernández, M.-V., Lameiras-Fernández, M., Rodríguez-Castro, Y., \& Vallejo-Medina, P. (2013). Bullying among Spanish secondary education students: The role of gender traits, sexism, and homophobia. Journal of Interpersonal Violence, 28(14), 2915-2940. https://doi.org/10.1177/0886260513488695

Carvalhosa, S. F., Moleiro, C., \& Sales, C. (2009). A situação do bullying nas escolas portuguesas. Interacções, 146(13), 125-146.

Carver, C. S. \& Scheier, M. F. (2002). Optimism. In C. R. Snyder \& S. J. Lopez (Eds.), The Handbook of Positive Psychology (pp. 231-243). New York: Oxford University Press.

Carver, C. S. (1997). You want to measure coping but your protocol's too long: Consider the Brief COPE. International Journal of Behavioral Medicine, 4(1), 92-100. https://doi.org/10.1207/s15327558ijbm0401_6

Carver, C. S., Scheier, M. F., \& Weintraub, J. K. (1989). Assessing coping strategies: A theoretically based approach. Journal of Personality and Social Psychology, 56(2), 267-283. https://doi.org/10.1037/0022-3514.56.2.267

Carver, C., \& Connor-Smith, J. (2010). Personality and coping. Annual Review of Psychology, 61, 679-704. https://doi.org/10.1146/annurev.psych.093008.100352

Catterson, J., \& Hunter, S. C. (2010). Cognitive mediators of the effect of peer victimization on loneliness. The British Journal of Educational Psychology, 80, 403-416. https://doi.org/10.1348/000709909X481274

Coimbra, S. M. (2008). Estudo diferencial de auto-eficácia e resiliência na antecipação da vida adulta (Dissertação de Doutoramento não publicada). Faculdade de Psicologia e de Ciências da Educação da Universidade do Porto, Porto.

Coimbra, S., \& Fontaine, A. M. (2015). Resiliência e habilidades sociais: Reflexões conceituais e práticas para uma nova geração. In Z. A. P. Del Prette, A. B. Soares, C. S Pereira-Guizzo, M. F. Wagner, \& V. B. R. Leme (Eds.) Habilidades sociais: diálogos e intercâmbios sobre pesquisa e prática (pp. 186-220). Novo Hamburgo: Synopsys.

Collishaw, S., Pickles, A., Messer, J., Rutter, M., Shearer, C., \& Maughan, B. (2007). Resilience to adult psychopathology following childhood maltreatment: Evidence from a community sample. Child Abuse \& Neglect, 31(3), 211-229. https://doi.org/10.1016/j.chiabu.2007.02.004

Costa, P. T., \& McCrae, R. R. (2000). NEO-PI-R Manual Profissional. Lisboa: CEGOC.

Costa-Lopes, R., Vala, J., Pereira, C., \& Aguiar, P. (2008). A construção social das diferenças nas relações entre grupos sociais. In M. Villaverde, K. Wall, S. Aboim, \& F. C. Silva (Eds.), Itinerários: A Investigação nos 25 Anos do ICS (pp. 769-790). Lisboa: Imprensa de Ciências Sociais.

Cunha, A. C. R. (2014). Bullying: um estudo exploratório das práticas parentais e das atitudes relativas à diversidade de género e etnia na compreensão do fenómeno (Tese de Mestrado não publicada). Universidade do Minho, Braga. http://repositorium.sdum.uminho.pt/bitstream/1822/35858/1/Ana\%20Catarina\%20Rodrigues $\% 20$ da\%20Cunha.pdf

Davis, M. H. (1983). Measuring individual differences in empathy: Evidence for a multidimensional approach. Journal of Personality and Social Psychology, 44(1), 113-126. https://doi.org/10.1037/0022-3514.44.1.113

del Barrio, C.; Martín, E.; Montero, I.; Gutiérrez, H.; Barrios, A. \& Dios, M. J. (2008). Bullying and social exclusion in Spanish secondary schools: National trends from 1999 to 2006. International Journal of Clinical and Health Psychology, 8(3), 657-677.

Dessel, A. (2010). Prejudice in schools: Promotion of an inclusive culture and climate. Education and Urban Society, 42(4), 407-429. https://doi.org/10.1177/0013124510361852

Diego, M. A., Field, T. M., \& Sanders, C. E. (2003). Academic performance, popularity, and depression predict adolescent substance use. Adolescence, 38(149), 35-42. https://doi.org/10.1177/014662167700100306]1979-10129-001

Dovidio, J., Major, B., \& Crocker, J. (2000). Stigma: Introduction and overview. In T. Heatherton, R. Kleck, M. Hebl, \& J. Hull (Eds.), The social psychology of stigma (pp. 1-30). New York: The Guildford Press.

Dumont, M., \& Provost, M. A. (1999). Resilience in adolescents: Protective role of social support, coping strategies self-esteem, and social activities on experience of stress and depression. Journal of Youth and Adolescence, 28(3), 343-363. https://doi.org/10.1023/A:1021637011732

Eisenberg, M., \& Resnick, M. (2006). Suicidality among gay, lesbian and bisexual youth: the role of protective factors. Journal of Adolescent Health, 39(5), 662-668. https://doi.org/10.1016/j.jadohealth.2006.04.024

Elamé, E. (2013). Discriminatory bullying: A new intercultural challenge. Italia: Springer-Verlag. https://doi.org/10.1007/978-88-470-5235-2 
European Commission. (2015). Special Eurobarometer 437: Discrimination in the EU in 2015. Discrimination in the EU in 2015. https://doi.org/10.2838/325154

Fedewa, A. L., \& Ahn, S. (2011). The effects of bullying and peer victimization on sexual-minority and heterosexual youths: A quantitative meta-analysis of the literature. Journal of GLBT Family Studies, 7(4), 398-418. https://doi.org/10.1080/1550428X.2011.592968

Fergus, S., \& Zimmerman, M. A. (2005). Adolescent resilience: A framework for understanding healthy development in the face of risk. Annual Review of Public Health, 26, 399-419. https://doi.org/10.1146/annurev.publhealth.26.021304.144357

Ferguson, C. J. (2009). An effect size primer: A guide for clinicians and researchers. Professional Psychology: Research and Practice, 40(5), 532-538. https://doi.org/10.1037/a0015808

Foster, M. D. (2009). Perceiving pervasive discrimination over time: Implications for coping. Psychology of Women Quarterly, 33(2), 172-182. https://doi.org/10.1111/j.1471-6402.2009.01487.x

França, D. X., \& Monteiro, M. B. (2004). A expressão das formas indirectas de racismo na infância. Análise Psicológica, 22(4), 705-720.

Freire, I. P., Simão, A. M. V., \& Ferreira, A. S. (2006). 0 estudo da violência entre pares no 3o ciclo do ensino básico - um questionário aferido para a população escolar portuguesa. Revista Portuguesa de Educação, 19(3), 157-183.

Freitas, D. F. (2016). Resiliência perante a violência social: Perfis de ajustamento e mecanismos de proteção. (Dissertação de Doutoramento não publicada). Faculdade de Psicologia e Ciências da Educação da Universidade do Porto e Faculdade de Filosofia, Ciências e Letras de Ribeirão Preto da Universidade de São Paulo. Disponível em: https://repositorioaberto.up.pt/bitstream/10216/85740/2/150342.pdf

Freitas, D. F., Coimbra, S., Marturano, E. M. \& Fontaine, A. M. (2015). Adaptação da Escala de Discriminação Quotidiana para Jovens Portugueses. Psychology /Psicologia Reflexão e Crítica, 28(4), 708-717. https://doi.org/10.1590/1678-7153.201528408

Freitas, D. F., Coimbra, S., Marturano, E. M., Marques, S. C., Oliveira, J. E., \& Fontaine, A. M. (2017). Resilience in the face of peer victimisation and discrimination: The who, when and why in five patterns of adjustment. Journal of Adolescence, 59, 19-34. https://doi.org/10.1016/j.adolescence.2017.05.009

Freitas, D. F., D'Augelli, A. R., Coimbra, S., \& Fontaine, A. M. (2016). Discrimination and mental health among gay, lesbian, and bisexual youths in Portugal: The moderating role of family relationships and optimism. Journal of GLBT Family Studies, 12(1), 68-90. https://doi.org/10.1080/1550428X.2015.1070704

Fullchange, A., \& Furlong, M. J. (2016). An exploration of effects of bullying victimization from a complete mental health perspective. SAGE Open, 6(1), 1-12. https://doi.org/10.1177/2158244015623593

Furman, W., \& Buhrmester, D. (1985). Children's perceptions of the personal relationships in their social networks. Developmental Psychology, 21(6), 1016-1024. https://doi.org/10.1037//00121649.21.6.1016

Furman, W., \& Buhrmester, D. (1992). Age and sex differences in perceptions of networks of personal relationships. Child Development, 63, 103-115. https://doi.org/10.1111/j.14678624.1992.tb03599.x

Gaspar, T. T., Matos, M. G., Ribeiro, J. L., \& Leal, I. (2010). Qualidade de vida em crianças e adolescentes: Impacto da migração e estatuto socio-econômico. Brazilian Journal of Health, 1(1), 80-92.

Gaspar, T., Gonçalves, A., Ramos, V., \& Matos, M. G. (2006). Desvantagem socio-económica, etnicidade e consumo de álcool na adolescência. Análise Psicológica, 24(4), 495-508.

Gato, J., Carneiro, N. S., \& Fontaine, A. M. (2011). Contributo para uma revisitação histórica e crítica do preconceito contra as pessoas não heterossexuais. Crítica e Sociedade: Revista de Cultura Política, 1(1), 139-167.

Gaylord-Harden, N. K., \& Cunningham, J. A. (2009). The impact of racial discrimination and coping strategies on internalizing symptoms in African American youth. Journal of Youth and Adolescence, 38(4), 532-543. https://doi.org/10.1007/s10964-008-9377-5

Gradinger, P., \& Strohmeier, D. (2015, setembro). Discrimination in austrian schools: Prevalence of multiple forms of discriminations and associated resiliences and risks. In S. Russell (Chair), Discriminatory bullying and adolescent adjustment: Cross-national evidence. Symposium conducted at the $17^{\text {th }}$ European Conference on Developmental Psychology. Braga, Portugal.

Graham, P., \& Rutter, M. (1973). Psychiatric disorder in the young adolescent: A follow-up study. Proceedings of the Royal Society of Medicine, 66(12), 1226-1229.

Graham, S., \& Juvonen, J. (1998). Self-blame and peer victimization in middle school: an attributional analysis. Developmental Psychology, 34(3), 587-599. https://doi.org/10.1037/0012-1649.34.3.587 
Hatzenbuehler, M. L. (2009). How does sexual minority stigma "get under the skin"? A psychological mediation framework. Psychological Bulletin, 135(5), 707-730. https://doi.org/10.1037/a0016441

Hawker, D. S. J., \& Boulton, M. J. (2000). Twenty years' research on peer victimization and psychosocial maladjustment: A meta-analytic review of cross-sectional studies. Journal of Child Psychology and Psychiatry, 41(4), 441-455. https://doi.org/10.1111/1469-7610.00629

Hawker, D. S. J., \& Boulton, M. J. (2001). Subtypes of peer harassment and their correlates: A social dominance perspective. In J. Juvonen \& S. Graham (Eds.) Peer harassment in school: The plight of the vulnerable and victimized (pp. 378-397). New York: The Guilford Press

Hemphill, S. A., Tollit, M., \& Herrenkohl, T. I. (2014). Protective factors against the impact of school bullying perpetration and victimization on young adult externalizing and internalizing problems. Journal of School Violence, 13(1), 125-145. https://doi.org/10.1080/15388220.2013.844072

Herman-Stahl, M., \& Petersen, A. C. (1996). The protective role of coping and social resources for depressive symptoms among young adolescents. Journal of Youth and Adolescence, 25(6), 733-753. https://doi.org/10.1007/BF01537451

Hunter, S. C., Boyle, J. M. E., \& Warden, D. (2007). Perceptions and correlates of peer-victimization and bullying. British Journal of Educational Psychology, 77(4), 797-810. https://doi.org/10.1348/000709906X171046

Hunter, S. C., Durkin, K., Heim, D., Howe, C., \& Bergin, D. (2010). Psychosocial mediators and moderators of the effect of peer-victimization upon depressive symptomatology. Journal of Child Psychology and Psychiatry, 51(10), 1141-1149. https://doi.org/10.1111/j.1469-7610.2010.02253.x

Juang, L., \& Alvarez, A. (2010). Discrimination and adjustment among chinese american adolescents: Family conflict and family cohesion as vulnerability and protective factors. American Journal of Public Health, 100(12), 2403-2409. https://doi.org/10.2105/AJPH.2009.185959

Katz-Wise, S. L., \& Hyde, J. S. (2012). Victimization experiences of lesbian, gay, and bisexual individuals: A $\begin{array}{lllll}\text { meta-analysis. Journal of } & \text { Sex }\end{array}$ https://doi.org/10.1080/00224499.2011.637247

Kaysen, D. L., Kulesza, M., Balsam, K. F., Rhew, I. C., Blayney, J. A., Lehavot, K., \& Hughes, T. L. (2014). Coping as a mediator of internalized homophobia and psychological distress among young adult sexual minority women. Psychology of Sexual Orientation and Gender Diversity, 1(3), 225-233. https://doi.org/10.1037/sgd0000045

Kochenderfer, B. J., \& Ladd, G. W. (1996). Peer victimization: Cause or consequence of school maladjustment? Child Development, 67(4), 1305. https://doi.org/10.2307/1131701

Krieger, N. (1999). Embodying inequality: A review of concepts, measures, and methods for studying health consequences of discrimination. International Journal of Health Services, 29(2), 295-352. https://doi.org/10.2190/M11W-VWXE-KQM9-G97Q

Kristensen, C. H., Leon, J. S., D'Incao, D. B., \& Dell'Aglio, D. D. (2004). Análise da frequência e do impacto de eventos estressores em uma amostra de adolescentes. Interação Em Psicologia, 8(1), 45-55. Disponível em: http://ojs.c3sl.ufpr.br/ojs2/index.php/psicologia/article/viewArticle/3238

Laranjeira, C. A. (2008). Tradução e validação portuguesa do Revised Life Orientation Test (LOT-R). Universitas Psychologica, 7(2), 469-476.

Laursen, B., \& Collins, W. A. (2009). Parent-child relationships during adolescence. In R. M. Lerner \& L. Steinberg (Eds.), Handbook of Adolescent Psychology (Vol. 2, pp. 3-42). Hoboken, NJ, USA: John Wiley \& Sons, Inc. https://doi.org/10.1002/9780470479193.adlpsy002002

Lempers, J. D., \& Clark-Lempers, D. S. (1992). Young, middle, and late adolescents' comparisons of the functional importance of five significant relationships. Journal of Youth and Adolescence, 21(1), 5396. https://doi.org/10.1007/BF01536983

Lima, M. P., \& Simões, A. (2006). Inventário de personalidade NEO revisto (NEO-PI-R). In M. M. Gonçalves, L. Almeida \& M. R. Simões (Eds.) Avaliação psicológica: Instrumentos validados para a população portuguesa (pp. 21-36). Coimbra: Quarteto.

Limpo, T., Alves, R. A., \& Castro, S. L. (2010). Medir a empatia: Adaptação portuguesa do Índice de Reactividade Interpessoal. Laboratório de Psicologia, 8(2), 171-184.

Luthar, S. S., Cicchetti, D., \& Becker, B. (2000). The construct of resilience: A critical evaluation and guidelines for future work. Child Development, 71(3), 543-62. https://doi.org/10.1111/14678624.00164

Marôco, J. (2011). Análise estatística com o SPSS Statistics (5a Ed.). Pêro Pinheiro, Portugal: Report Number.

Marques, S. C., Pais-ribeiro, J. L., \& Lopez, S. J. (2011). Use of the "Mental Health Inventory - 5" with Portuguese 10-15 years old. The Spanish Journal of Psychology, 14(1), 478-485. https://doi.org/10.5209/rev_SJOP.2011.v14.n1.43 
Masten, A. S. (2001). Ordinary magic: Resilience processes in development. American Psychologist, 56(3), 227-238. https://doi.org/10.1037/0003-066X.56.3.227

Masten, A. S., \& Tellegen, A. (2012). Resilience in developmental psychopathology: Contributions of the Project Competence Longitudinal Study. Development and Psychopathology, 24(2), 345-361. https://doi.org/10.1017/S095457941200003X

Masten, A. S., Hubbard, J. J., Gest, S. D., Tellegen, A., Garmezy, N., \& Ramirez, M. (1999). Competence in the context of adversity: pathways to resilience and maladaptation from childhood to late adolescence. Development and Psychopathology, 143-169. https://doi.org/10.1017/S0954579499001996

Masten, A.S., \& Reed, M.G. (2002). Resilience in development. In C.R. Snyder \& S. J. Lopez (Eds.), The Handbook of Positive Psychology (pp. 74-88). New York: Oxford University Press.

Masten, A.S., \& Wright, M. O. (2010). Resilience over the lifespan: Developmental Perspectives on Resistance, Recovery, and Transformation. In J. Reich, A. Zautra \& J. Hall (Eds.), Handbook of Adult Resilience (pp. 213-237). New York: Guilford Press

Matos, M. G., Gonçalves, A., \& Gaspar, T. (2004). Adolescentes estrangeiros em Portugal: Uma questão de saúde. Psicologia, Saúde \& Doenças, 5(1), 75-85.

Matos, M. G., Simões, C., \& Gaspar, T. (2009). Violência entre pares no contexto escolar em Portugal, nos últimos 10 anos. Interacções, 5(13), 98-124.

McVie, S. (2014). The impact of bullying perpetration and victimization on later violence and psychological distress: A study of resilience among a Scottish youth cohort. Journal of School Violence, 13(1), 39-58. https://doi.org/10.1080/15388220.2013.841586

Mendonça, M., \& Fontaine, A. M. (2013). Late nest leaving in Portugal: Its effects on individuation and parent-child relationships. Emerging Adulthood, 1(3), 233-244. https://doi.org/10.1177/2167696813481773

Murdock, T. B., \& Bolch, M. B. (2005). Risk and protective factors for poor school adjustment in lesbian, gay, and bisexual (LGB) high school youth: Variable and person-centered analyses. Psychology in the Schools, 42(2), 159-172. https://doi.org/10.1002/pits.20054

Mynard, H., \& Joseph, S. (2000). Development of the Multidimensional Peer-Victimization Scale. Aggressive Behavior, 26(2), 169-178. https://doi.org/10.1002/(SICI)10982337(2000)26:2<169::AID-AB3>3.0.CO;2-A

Neto, F. (2009). Predictors of mental health among adolescents from immigrant families in Portugal. Journal of Family Psychology, 23(3), 375-85. https://doi.org/10.1037/a0015831

Neto, F. (2010a). Portugal intercultural: Aculturação e adapatação de jovens de origem imigrante. Oliveira de Azeméis: Livpsi.

Neto, F. (2010b). Predictors of adaptation among adolescents from immigrant families in Portugal. Journal of Comparative Family Studies, 41(3), 437-454.

Nolen-Hoeksema, S., \& Girgus, J. S. (1994). The emergence of gender differences in depression during adolescence. Psychological Bulletin, 115(3), 424-443. https://doi.org/10.1037/00332909.115.3.424

Ortega, R., Elipe, P., Mora-Merchán, J. A., Calmaestra, J., \& Vega, E. (2009). The emotional impact on victims of traditional bullying and cyberbullying. Zeitschrift Für Psychologie / Journal of Psychology, 217(4), 197-204. https://doi.org/10.1027/0044-3409.217.4.197

Paradies, Y., Ben, J., Denson, N., Elias, A., Priest, N., Pieterse, A., ... Gee, G. (2015). Racism as a determinant of health: A systematic review and meta-analysis. PLOS ONE, 10(9), 1-48. https://doi.org/10.1371/journal.pone.0138511

Pascoe, E., \& Richman, L. (2009). Perceived discrimination and health: a meta-analytic review. Psychological Bulletin, 135(4), 531-54. https://doi.org/10.1037/a0016059

Pearson, J., \& Wilkinson, L. (2013). Family relationships and adolescent well-being: Are families equally protective for same-sex attracted youth? Journal of Youth and Adolescence, 42(3), 376-393. https://doi.org/10.1007/s10964-012-9865-5

Pereira, B., Mendonça, D., Neto, C., Valente, L., \& Smith, P. K. (2004). Bullying in Portuguese schools. School Psychology International, 25(2), 241-254. https://doi.org/10.1177/0143034304043690

Quental, C., \& Veiga, F. (2010). Maltrato entre alunos do ensino básico: Análise em função do rendimento e da disrupção escolar. Actas do XVII Colóquio AFIRSE: A escola e o mundo do trabalho (pp. 232-241). Disponível em: http://hdl.handle.net/10451/5450

Reijntjes, A., Kamphuis, J. H., Prinzie, P., \& Telch, M. J. (2010). Peer victimization and internalizing problems in children: A meta-analysis of longitudinal studies. Child Abuse and Neglect, 34(4), 244252. https://doi.org/10.1016/j.chiabu.2009.07.009

Reijntjes, A., Kamphuis, J. H., Prinzie, P., Boelen, P. A., Van Der Schoot, M., \& Telch, M. J. (2011). Prospective 
linkages between peer victimization and externalizing problems in children: A meta-analysis. Aggressive Behavior, 37(3), 215-222. https://doi.org/10.1002/ab.20374

Ribeiro, J. P. (2001). Mental Health Inventory: Um estudo de adaptação à população Portuguesa. Psicologia, Saúde \& Doenças, 2(1), 77-99.

Ribeiro, J. P., \& Rodrigues, A. P. (2004). Questões acerca do coping: a propósito do estudo de adaptação do Brief COPE. Psicologia, Saúde \& Doenças, 5(1), 3-15.

Richman, L. S., \& Leary, M. R. (2009). Reactions to discrimination, stigmatization, ostracism, and other forms of interpersonal rejection: A multimotive model. Psychological Review, 116(2), 365-83. https://doi.org/10.1037/a0015250

Rivers, I., \& Cowie, H. (2006). Bullying and homophobia in UK schools: A perspective on factors affecting resilience and recovery. Journal of Gay \& Lesbian Issues in Education, 3(4), 11-43. https://doi.org/10.1300/J367v03n04_03

Rodrigues, L., Brás, A., Cunha, C., Petiz, J. P., \& Nogueira, C. (2015). Teachers' discourses on young lesbians in the portuguese school context. Actualidades Investigativas En Educación, 15(2), 1-19. https://doi.org/10.15517/aie.v15i2.18535

Rose, A. J., \& Rudolph, K. D. (2006). A review of sex differences in peer relationship processes: Potential trade-offs for the emotional and behavioral development of girls and boys. Psychological Assessment, 132(1), 98-131. https://doi.org/10.1037/0033-2909.132.1.98

Russell, S. T., Sinclair, K. O., Poteat, V. P., \& Koenig, B. W. (2012). Adolescent health and harassment based on discriminatory bias. American Journal of Public Health, 102(3), 493-495. https://doi.org/10.2105/AJPH.2011.300430

Rutter, M. (2001). Psychosocial adversity: Risk, resilience and recovery. In J. M. Richman \& M. W. Fraser (Eds.) The context of youth violence: Resilience, risk, and protection (pp. 13-41). Westport: Praeger

Rutter, M. (2005). Multiple meanings of a developmental perspective on psychopathology. European $\begin{array}{llll}\text { Journal of Developmental } & \text { Psychology, 2(3), 221-252. }\end{array}$ https://doi.org/10.1080/17405620500237706

Rutter, M. (2013). Annual Research Review: Resilience - clinical implications. Journal of Child Psychology and Psychiatry, 54(4), 474-487. https://doi.org/10.1111/j.1469-7610.2012.02615.x

Rutter, M., Caspi, A., \& Moffitt, T. E. (2003). Using sex differences in psychopathology to study causal mechanisms: unifying issues and research strategies. Journal of Child Psychology and Psychiatry, 44(8), 1092-1115. https://doi.org/10.1111/1469-7610.00194

Ryan, C., Russell, S., Huebner, D., Diaz, R., \& Sanchez, J. (2010). Family acceptance in adolescence and the health of LGBT young adults. Journal of Child and Adolescent Psychiatric Nursing, 23(4), 205-13. https://doi.org/10.1111/j.1744-6171.2010.00246.x

Saewyc, E. M. (2011). Research on adolescent sexual orientation: Development, health disparities, stigma, and resilience. Journal of Research on Adolescence, 21(1), 256-272. https://doi.org/10.1111/j.1532-7795.2010.00727.x

Schacter, H. L., White, S. J., Chang, V. Y., \& Juvonen, J. (2015). “Why me?”: Characterological self-blame and continued victimization in the first year of middle school. Journal of Clinical Child \& Adolescent Psychology, 44(3), 446-455. https://doi.org/10.1080/15374416.2013.865194

Scheier, M. F., \& Carver, C. S. (1985). Optimism, coping, and health: assessment and implications of generalized outcome expectancies. Health Psychology, 4(3), 219-247. https://doi.org/10.1037/0278-6133.4.3.219

Schmitt, M. T., Branscombe, N. R., Postmes, T., \& Garcia, A. (2014). The consequences of perceived discrimination for psychological well-Being: A meta-analytic review. Psychological Bulletin, 140(4), 921-948. https://doi.org/10.1037/a0035754

Shiner, R. L., \& Masten, A. S. (2012). Childhood personality as a harbinger of competence and resilience in adulthood. Development and Psychopathology, 24(2), 507-528. https://doi.org/10.1017/S0954579412000120

Smetana, J. G., Campione-Barr, N., \& Metzger, A. (2006). Adolescent development in interpersonal and societal contexts. Annual Review of Psychology, 57, 255-84. https://doi.org/10.1146/annurev.psych.57.102904.190124

Strohmeier, D., Kärnä, A., \& Salmivalli, C. (2011). Intrapersonal and interpersonal risk factors for peer victimization in immigrant youth in Finland. Developmental Psychology, 47(1), 248-258. https://doi.org/10.1037/a0020785

Swearer, S. M., Espelage, D. L., Vaillancourt, T., \& Hymel, S. (2010). What can be done about school bullying?: Linking research to educational practice. Educational Researcher, 39(1), 38-47. https://doi.org/10.3102/0013189X09357622

Swearer, S. M., \& Hymel, S. (2015). Understanding the psychology of bullying: Moving toward a social- 
ecological diathesis-stress. American Psychologist, 70(4), 344-353. https://doi.org/10.1037/a0038929

Szalacha, L. A., Erkut, S., Coll, C. G., Fields, J. P., Alarcón, O., \& Ceder, I. (2003). Perceived Discrimination and Resilience. In S. S. Luthar (Ed.), Resilience and Vulnerability: Adaptation in the Context of Childhood Adversities (pp. 414-435). New York: Cambridge University Press.

Takizawa, R., Maughan, B., \& Arseneault, L. (2014). Adult health outcomes of childhood bullying victimization: Evidence from a five-decade longitudinal British birth cohort. American Journal of Psychiatry, 171(7), 777-784. https://doi.org/10.1176/appi.ajp.2014.13101401

Thornberg, R. (2010). Schoolchildren's social representations on bullying causes. Psychology in the Schools, 74(4), 311-327. https://doi.org/10.1002/pits.20472

Ttofi, M. M., Bowes, L., Farrington, D. P., \& Lösel, F. (2014). Protective factors interrupting the continuity from school bullying to later internalizing and externalizing problems: A systematic review of prospective longitudinal studies. Journal of School Violence, 13(1), 5-38. https://doi.org/10.1080/15388220.2013.857345

Ungar, M. (2000). The myth of peer pressure. Adolescence, 35(137), 167-180.

Ungar, M. (2004). A constructionist discourse on resilience: Multiple contexts, multiple realities among atrisk children and youth. Youth \& Society, 35(3), 341-365. https://doi.org/10.1177/0044118X03257030

Ungar, M. (2011). The social ecology of resilience: Addressing contextual and cultural ambiguity of a nascent construct. American Journal of Orthopsychiatry, 81(1), 1-17. https://doi.org/10.1111/j.1939-0025.2010.01067.x

Ungar, M. (2013). Resilience, trauma, context, and culture. Trauma, Violence, \& Abuse, 14(3), 255-266. https://doi.org/10.1177/1524838013487805

Vanderbilt-Adriance, E., \& Shaw, D. S. (2008). Conceptualizing and re-evaluating resilience across levels of risk, time, and domains of competence. Clinical Child and Family Psychology Review, 11(1-2), 30-58. https://doi.org/10.1007/s10567-008-0031-2

Vassallo, S., Edwards, B., Renda, J., \& Olsson, C. A. (2014). Bullying in early adolescence and antisocial behavior and depression six years later: What are the protective factors? Journal of School Violence, 13(1), 100-124. https://doi.org/10.1080/15388220.2013.840643

Veiga, F. H. (2007). Adaptação da "Multidimensional Peer Victimization Scale" para Portugal. XIII Conferência Internacional sobre "Avaliação Psicológica: Formas e Contextos." Braga, Portugal. Disponível em: http://repositorio.ul.pt/jspui/bitstream/10451/5815/1/Adapta\%C3\%A7\%C3\%A3o\%20da\%20M ultidimensional\%20Peer\%20Victimization\%20Scale\%20para\%20Portugal.pdf

Veit, C. T., \& Ware, J. E. (1983). The structure of psychological distress and well-being in general populations. Journal of Consulting and Clinical Psychology, 51(5), 730-742. https://doi.org/10.1037/0022-006X.51.5.730

Walsh, F. (2002). A family resilience framework: innovative practice applications. Family Relations, 51, 130-137. https://doi.org/10.1111/j.1741-3729.2002.00130.x

Walsh, F. (2003). Family resilience: A framework for clinical practice. Family Process, 42(1), 1-18. https://doi.org/10.1111/j.1545-5300.2003.00001.x

Werner, E. E. (1993). Risk, resilience, and recovery: Perspectives from the Kauai Longitudinal Study. Development and Psychopathology, 5(4), 503-515. https://doi.org/10.1017/S095457940000612X

WHO (2014). Mental health: a state of well-being. Consultado em Fevereiro de 2015 em: http://www.who.int/features/factfiles/mental_health/en/

Williams, D. R., Yu, Y., Jackson, J. S., \& Anderson, N. B. (1997). Racial differences in physical and mental health. Journal of Health Psychology, 2(3), 335-351. https://doi.org/10.1177/1359105397002

Historial do artigo

Recebido $\quad 01 / 05 / 2016$

Aceite 27/07/2017

Publicado 11/2017 
Mecanismos de proteção perante a violência social

Está página encontra-se propositadamente em branco 